\title{
Task-dependent and independent synchronous activity of monkey hippocampal neurons in real and virtual translocation
}

\author{
Etsuro Hori', Eiichi Tabuchi', Nobuhisa Matsumura' ${ }^{1}$ Taketoshi Ono ${ }^{3}$ and Hisao Nishijo ${ }^{\text {* }}$ \\ System Emotional Science, Graduate School of Medicine and Pharmaceutical Sciences, University of Toyama, Toyama, Japan \\ 2 Department of Neurosurgery, Graduate School of Medicine and Pharmaceutical Sciences, University of Toyama, Toyama, Japan \\ 3 Judo Neurophysiotherapy, Graduate School of Medicine and Pharmaceutical Sciences, University of Toyama, Toyama, Japan
}

\section{Edited by:}

Carlos Tomaz, University of Brasilia,

Brazil

\section{Reviewed by:}

Carlos Tomaz, University of Brasilia, Brazil

Patrizia Campolongo, Università degli Studi di Roma La Sapienza, Italy

${ }^{*}$ Correspondence:

Hisao Nishijo, System Emotional Science, University of Toyama, Sugitani 2630, Toyama 930-0194, Japan. e-mail:nishijo@med.u-toyama.ac.jp
Previous neurophysiological and behavioral studies relate hippocampal functions to place learning and memory, and encoding of task (or context)-specific information. Encoding of both taskspecific information and own location is essential for episodic memory and for animals to navigate to reward-related places. It is suggested that different neural circuits with different assemblies of different hippocampal neurons are created in different environments or behavioral contexts for the hippocampal formation (HF) to encode and retrieve episodic memory. To investigate whether synchronous activity of hippocampal neurons, suggesting functional connectivity between those neurons, is task and position dependent, multiple single unit activities were recorded during performance of real and virtual translocation (VT) tasks. The monkey moved to one of four reward areas by driving a cab (real translocation) or by moving a pointer on a monitor. Of 163 neuron pairs, significant peaks in cross-correlograms (CCGs) were observed in 98 pairs. Most CCGs had positive peaks within $50 \mathrm{~ms}$. Task-dependent cross-correlations (CCRs) were observed in $44 \%$ of the neuron pairs, and similarly observed in both the real andVT tasks. These CCRs were frequently observed in pyramidal vs. pyramidal neuron pairs with positive peak and peak shift. However, no consistent patterns of peak polarity, peak shift, and neuronal types were seen in task-independent CCRs. There was no significant difference in frequency of CCG peaks between real and VT tasks. These results suggest that the task-dependent information may be encoded by interaction among pyramidal neurons, and the common information across tasks may be encoded by interaction among pyramidal neurons and interneurons in the HF. These neuronal populations could provide a neural basis for episodic memory to disambiguously guide animals to places associated with reward in different situations.

Keywords: place cell, cross-correlation, auto-correlation, reference frame, episodic memory

\section{INTRODUCTION}

It has been suggested that food-storing birds use memory to retrieve stored food and have a larger hippocampal formation (HF; Clayton, 1995). The HF is important for encoding the association of taste with context (Gallo, 2005; Ho et al., 2011). The HF also plays an important role in a place-reward association in monkeys (Hampton et al., 2004), and monkey HF neurons differentially responded in a place-reward association task (Rolls and Xiang, 2005). Consistently, the HF is involved in conditioned place preference in which animals learned to prefer a chamber where a conditioned stimulus was associated with sucrose solution (Ito et al., 2006, 2008). These results suggest that the HF is important in mapping the location of rewards, and in guiding animals to places previously associated with reward.

Hippocampal formation neurons are active when the animal occupies a specific location (O'Keefe and Dostrovsky, 1971; McNaughton et al., 1983; Eichenbaum et al., 1987; Muller and Kubie, 1987). Unit-recording studies demonstrate that primate $\mathrm{HF}$ and parahippocampal gyrus $(\mathrm{PH})$ neurons respond to spatial cues (O’Mara et al., 1994; Eifuku et al., 1995; Suzuki et al., 1997). Recent neuropsychological studies in humans have demonstrated a pivotal role of the medial temporal lobe including the $\mathrm{HF}$ and $\mathrm{PH}$ and its related areas in allocentric spatial information processing (Maguire et al., 1996a; Abrahams et al., 1997; Takahashi et al., 1997). Consistent with these studies, positron emission tomography (PET) and functional magnetic resonance imaging (fMRI) have revealed blood flow increase in the HF and PH during various types of spatial tasks using a real or realistic virtual environment in normal humans (Aguirre et al., 1996; Ghaem et al., 1997; Maguire et al., 1997; Mellet et al., 2000).

It has been reported that activity of HF neurons is affected by the contexts of the tasks (Nishijo et al., 1993; Skaggs and McNaughton, 1998; Matsumura et al., 1999; Wood et al., 2000). This suggests that the HF encodes different reference frames; this might constitute a neural basis of episodic memory (Nishijo et al., 1993; Redish and Touretzky, 1997; Samsonovich and McNaughton, 1997; Skaggs and McNaughton, 1998). To understand the relationship between spatial selectivity and taskdependency of HF neurons, we have investigated the neuronal activities in allocentric space in real and virtual translocation (VT), in which the monkey in a movable cab could freely move toward a destination by manipulating a joystick (Ono et al., 1993; Nishijo et al., 1997; Matsumura et al., 1999; Hori et al., 2003). The results indicated that activity of $40 \%$ of monkey HF neurons was 
location-selective, and that most neurons showed task-dependent responses. This suggests that the HF is crucial in allocentric information processing, and that the HF may encode contexts of reference frames. Furthermore, the HF neurons display different spatial responses depending on motivational states (hunger or thirst) and reward (food or water; Kennedy and Shapiro, 2009), and on movement trajectories (Frank et al., 2000; Wood et al., 2000; Dayawansa et al., 2006). These results suggest that the HF forms multiple representations of the outer world depending on these factors. Previous theoretical studies proposed that the HF represented the external world by a reference frame (Redish and Touretzky, 1997) or chart (Samsonovich and McNaughton, 1997) system in which different neural circuits with different assemblies of different HF neurons were created in different environments or behavioral contexts.

To investigate neuronal ensemble activity with functional connectivity, cross-correlation (CCR) has been applied in studies of HF neurons using awake rats (Sakurai, 1996; Tabuchi et al., 2000; Hirase et al., 2001; Takahashi and Sakurai, 2009). Activity patterns of neuronal ensembles and degree of temporal correlation in CCRs were correlated to behavioral performance or behavioral states in the rodent striatum (Jog et al., 1999) and HF (Hirase et al., 2001). We hypothesized that the neuronal networks in the HF encode contexts of reference frames, by which appropriate behaviors in given situations might be chosen, i.e., different neural circuits in the HF might encode respective contexts. In the present study, to determine whether the functional neuronal connectivity among the HF neurons changes across different tasks, we reanalyzed the data set of the monkey HF neuronal activity during performance of the four different real and VT tasks (Matsumura et al., 1999) using a shift register difference CCR analysis.

\section{MATERIALS AND METHODS ANIMALS AND EXPERIMENTAL APPARATUS}

Two adult monkeys (Macaca fuscata), weighing 4.2 and $5.6 \mathrm{~kg}$, were used. The experimental protocol was described in detail in the previous paper (Matsumura et al., 1999). The monkeys were treated in strict compliance with the United States Public Health Service Policy on Human Care and Use of Laboratory Animals and the National Institutes of Health Guide for the Care and Use of Laboratory Animals, and the Guideline for the Care and Use of Laboratory Animals in our university (University of Toyama), and all efforts were made to minimize the number of animals used and their suffering.

The monkey was restrained painlessly in a stereotaxic apparatus by a surgically fixed head holder and sat in a $0.7 \mathrm{~m} \times 0.8 \mathrm{~m} \times 0.85 \mathrm{~m}$ high cab, which could freely move in a $2.5 \mathrm{~m} \times 2.5 \mathrm{~m}$ experimental field located in a $5.0 \mathrm{~m} \times 6.0 \mathrm{~m}$ room. The front wall of the cab was made of transparent Plexiglass and the rear wall consisted of a steel plate with two speakers. In the upper part of the front wall there was a color liquid crystal display (LCD) monitor (diagonal, $26 \mathrm{~cm}$ ) with a resolution of $640 \times 480$ pixels (LMD-1040XC, Sony), $25 \mathrm{~cm}$ from the monkey, on which visual stimuli were displayed. The lower part of the front wall contained a joystick used to move the cab in the experimental field and/or a pointer (an yellow circle with a radius of $3 \mathrm{~mm}$ ) on the LCD monitor. The cab and/or the pointer could be moved at a constant velocity $(\mathrm{cab}, 3 \mathrm{~cm} / \mathrm{s}$ in the experimental field; pointer, $2.5 \mathrm{~mm} / \mathrm{s}$ on the LCD monitor) in all four directions during the continuous manipulation of the joystick by the monkey.

The room, illuminated at 140 lux, contained several readily identified landmarks (sink, stereomicroscope, refrigerator, etc.; Figure 1Aa). The monkey sat in a chair within the cab and could see these visual landmarks in the experimental room, which were available to identify its position in the experimental field. The monkey performed four behavioral tasks under the guidance of visual stimuli on the LCD monitor and auditory stimuli from the two speakers. The tasks was to move the cab to one of four reward areas (target areas with a radius of $20 \mathrm{~cm}$ ) in the experimental field [real translocation (RT) task], or to move the pointer to one of four reward areas (target circles with a radius of $16 \mathrm{~mm}$ ) on the LCD monitor VT task by manipulating the joystick. The twodimensional space on the LCD monitor was proportional to the experimental field (1:12.61; Figure 1Ab).

\section{BEHAVIORAL PARADIGMS}

There were four kinds of behavioral tasks based on a combination of cab movements, indication of the pointer, and a target circle on the LCD monitor as described below. Time charts of the four behavioral tasks are shown in Figure 1B. More detailed methods were described previously (Matsumura et al., 1999).

\section{REAL TRANSLOCATION TASK WITHOUT A POINTER UNDER CONTINUOUS PRESENTATION OF A TARGET CIRCLE ON THE LCD MONITOR (RT/TC TASK)}

After the cab was placed at a starting point, the task was initiated by a 1 -s presentation of a warning tone $(1300 \mathrm{~Hz})$, followed by presentation of a $20 \mathrm{~cm} \times 20 \mathrm{~cm}$ blue square frame on the LCD monitor, which corresponded to the $2.5 \mathrm{~m} \times 2.5 \mathrm{~m}$ experimental field (warning phase). In this task, the blue square frame worked as a kind of a map in the experimental field in a scale ratio of 1:12.61 (Figure 1Ab). Then, a target circle (a red circle with a radius of $1.6 \mathrm{~cm}$ ) appeared at one of the four corners of the blue frame. Respective target areas in the experimental field (i.e., destination of the cab) corresponded to the four target circles on the LCD monitor. After $2 \mathrm{~s}$ presentation of the target circle (discrimination phase), the monkey manipulated the joystick to drive the cab toward the target area by recognizing its own location from the landmarks in the experimental field (manipulating/response phase). When the monkey arrived at the target area, a reward of approximately $6 \mathrm{ml}$ of orange juice was given for $3 \mathrm{~s}$ (reward phase). After delivery of a juice reward, the warning tone was stopped and the blue square frame and target circle disappeared. If the monkey failed to drive the cab to the target area within $120 \mathrm{~s}$, the trial was terminated and reward was withheld. At the end of each trial the cab was moved to the next starting point under a computer control. Time interval between trials was 30-60 s.

\section{REAL TRANSLOCATION TASK WITH A POINTER UNDER CONTINUOUS PRESENTATION OF A TARGET CIRCLE ON THE LCD MONITOR (RT/P-TC TASK)}

The task sequence and behavioral requirements were similar to those in the RT/TC task except that the pointer, indicating the location of the cab, was shown on the LCD monitor. Here, the monkey 
A

a. Experimental field

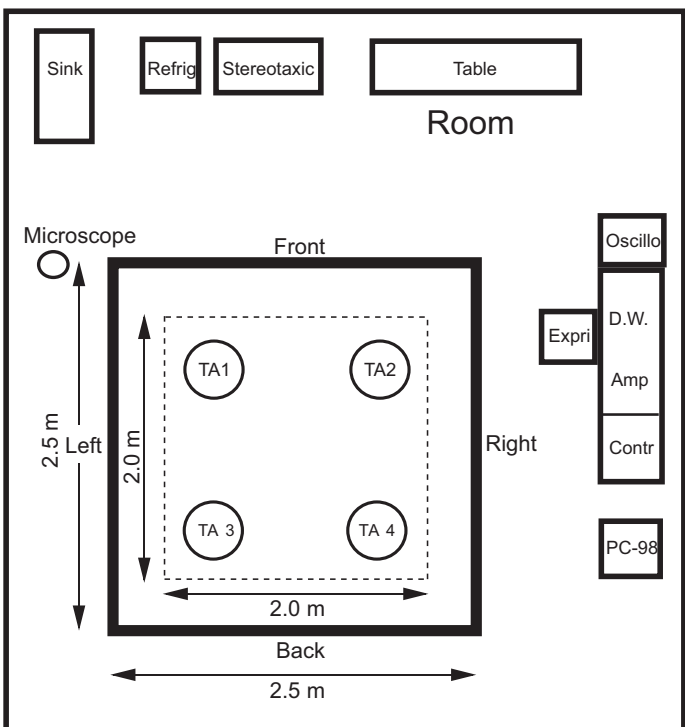

b. LCD monitor

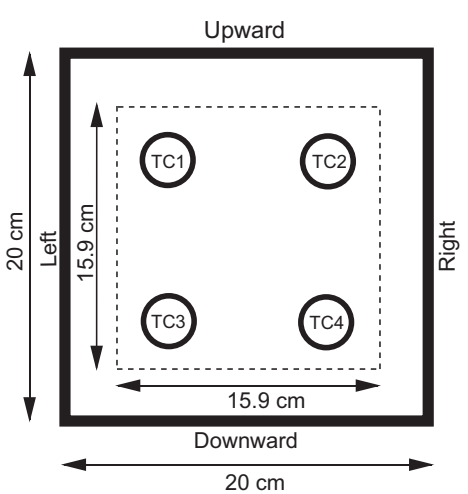

B

Warning Discrimination Manipulating/Response Reward

$\mathrm{RT} / \mathrm{TC}$
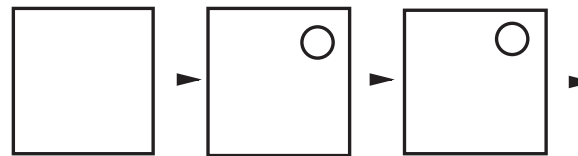

RT/P-TC
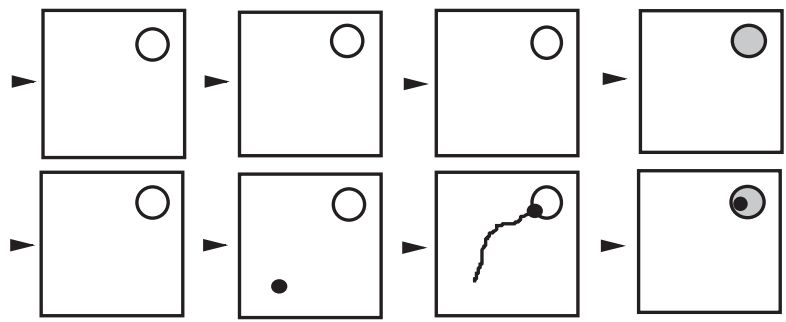

VT/P
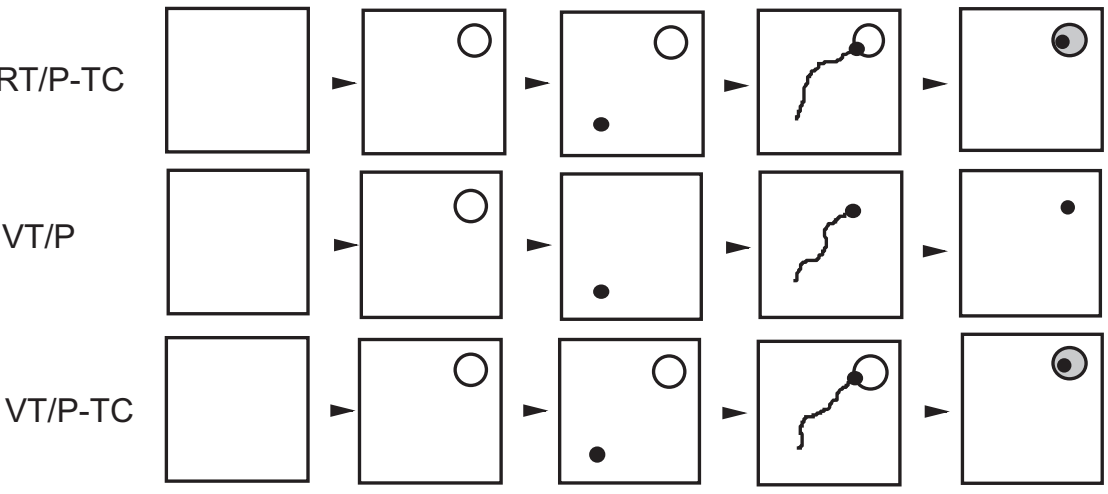

FIGURE 1 | Experimental set up (A) and task paradigms (B). (A) Spatial arrangements of room cues and target areas for the real translocation (RT) task (a) and those of target circles on the LCD monitor for the RT and virtual translocation (VT) tasks (b). In the RT tasks (a), the monkey moved the cab to one of four reward areas in the four corners of the experimental field [target area (TA) 1, 2, 3, or 4] by manipulating the joystick. Area in a thick line square, $2.5 \mathrm{~m} \times 2.5 \mathrm{~m}$ experimental field; area in a dotted line square, range of movement of a center of the cab where the monkey sat in a chair. Refrig, refrigerator; Stereotaxic, stereotaxic apparatus; Oscillo, oscilloscope; Expri, experimenter; D.W., microcomputer for Datawave; Amp, main amplifier; Contr, task controller; PC-98, microcomputer for monitoring movements of the cab and joystick. In the VT tasks (b) the monkey moved a pointer on the LCD monitor to one of four reward areas in the four corners of the LCD monitor [target circle (TC) 1, 2, 3, or 4] by manipulating the joystick. Area in a dotted line square, range of movement of the pointer on the LCD monitor. (B) Time chart of the four behavioral tasks. Each square indicates the frame which appeared on the color liquid crystal display (LCD) monitor. All four tasks were consisted of four phases; (1) warning phase: the warning tone and the frame were presented for $1 \mathrm{~s},(2)$ discrimination phase: a red target circle (radius, $15.9 \mathrm{~mm}$ ) appeared on the LCD monitor for $2 \mathrm{~s}$, (3) manipulating/response phase: a monkey manipulated a joystick to drive a cab or move a pointer on the LCD monitor, and (4) reward phase: approximate $6 \mathrm{ml}$ of orange juice was delivered for $3 \mathrm{~s}$. In the real translocation (RT) tasks, one of four target circles (TC), corresponding to one of four target areas in the experimental field, was presented on the LCD monitor. Then the monkey manipulated the joystick to drive the cab toward the indicated target area with (RT/P-TC task) or without (RT/TC task) guidance of a pointer (P), indicating location of the cab, on the monitor. In the virtual translocation (VT) tasks, the cab was located stationally throughout the trial. After one of the target circles was presented on the LCD monitor, the monkey manipulated the joystick to move a pointer toward the indicated location of the target circle without (VT/P task) or with (VT/P-TC task) presentation of the target circle during the manipulating/response phase. 
could use the LCD monitor as a map of the experimental field on which location of the monkey was indicated by the pointer. This is a similar situation to that for a car navigator, in which location of a car is indicated in a map on a LCD display. The task phases were same as those of the RT/TC task.

\section{VIRTUAL TRANSLOCATION TASK WITH A POINTER (VT/P TASK)}

The cab was stationary throughout the trial. A target circle was transiently presented on the LCD monitor only during the discrimination phase. Therefore, the monkey was required to memorize the location of the target circle during the discrimination phase, and to move the pointer to the location based on memory by manipulating the joystick. The task phases were same as those in the RT/TC task.

\section{VIRTUAL TRANSLOCATION TASK WITH A POINTER UNDER CONTINUOUS PRESENTATION OF A TARGET CIRCLE ON THE LCD MONITOR (VT/P-TC TASK)}

The task sequence and behavioral requirements were similar to those in the VT/P task except that a target circle was presented during the discrimination and manipulating/response phases. The task phases were same as those in the RT/TC task.

\section{CHARACTERISTICS OF EACH TASK}

These four tasks were so designed for behavioral requirement (i.e., joystick manipulation) and reward (juice) as to be similar across the tasks. However, different knowledge systems were assumed to be required to accomplish each task. It is noted that the pointer in the LCD monitor was not presented in the RT/TC task. In addition, the monkey always faced in the fixed direction, and consequently the same landmarks were seen in each trial. Therefore, the monkey had to judge its position based on the survey knowledge of the landmarks (Thorndyke and Hayes-Roth, 1982; Aguirre and D'Esposito, 1997) in the experimental room in the RT/TC task (i.e., relative spatial knowledge of place). Furthermore, the monkey was able to change its course flexibly during translocation when movement direction deviated from the destination (Matsumura et al., 1999). These support an idea that the monkey's behavior was based on a cognitive map in the brain (locale system), in which the spatial relationships of various landmarks are represented, rather than taxon systems where a set of stimulus-response (e.g., single landmarks to actions or movements) associations are represented (O'Keefe and Nadel, 1978). The situation in the RT/TC task corresponds to those in the previous human studies in which human subjects with temporal lobe lesions displayed deficits in topographical orientation such as way-finding from environmental video films (Maguire et al., 1996a), and in which cerebral blood flow in the HF of normal human subjects increased during learning of navigation routes from video films (Maguire et al., 1996b). In contrast, the monkey did not necessarily judge its position based on the cognitive maps in the RT/P-TC task since its position was indicated by the pointer on the LCD monitor. It is noted that behavioral requirements in the $\mathrm{RT} / \mathrm{P}-\mathrm{TC}$ task were similar to those in the RT/ TC tasks except this point.

In the VT tasks cognitive spatial information processing (information processing in the locale system) was not necessary since the pointer and/or a target circle were continuously presented on the monitor and the cab was located in a fixed place. The VT/P task required the monkey to memorize location of the target area on the monitor, so that this task was employed to test two-dimensional spatial short-term memory. Since locations of both the target area and the monkey or pointer were indicated in the RT/P-TC and VT/P-TC tasks, the situations in these two tasks correspond to a visible platform condition in a water maze paradigm for rodents in which animals with HF lesions performed normally (Morris et al., 1982). In spite of these differences among the four tasks, all these four tasks required to some extent spatial information processing.

\section{TRAINING AND SURGERY}

The monkey was initially trained to learn the VT/P-TC task. However, the monkey was required to move the pointer on the LCD monitor in fixed directions (i.e., forward-backward, leftward-rightward, or diagonally) by manipulating a joystick that had physical limitations of movements. It took about 2-3 months for the monkey to learn moving the pointer freely in all directions using a joystick without physical limitations. When the monkey learned to perform the VT/P-TC task with a criterion of $95 \%$ correct responses, it was then trained on the VT/P task. The monkey required about 3 months of training to reach $95 \%$ performance in the VT/P task. In the next stage, the monkey was trained to learn the RT/P-TC task. It learned the task very easily. After learning these three tasks with $95 \%$ performance, the monkey was trained in the RT/TC task. It took about 5 months of training for it to reach $95 \%$ performance. In the final stage, the monkey was well trained and performed all tasks for 3 weeks. The monkey was trained for $3 \mathrm{~h} /$ day and 5 day/week.

After completion of this training period, a head-restraining device (a U-shaped aluminum plate) was attached to the skull under aseptic condition and sodium pentobarbital anesthesia $(35 \mathrm{mg} / \mathrm{kg}$, i.m.). The plate was anchored with dental acrylic to stainless steel bolts inserted in keyhole slots in the skull. During the surgery, heart and respiratory functions and rectal temperature were monitored on a polygraph system (Nihon Kohden, Tokyo, Japan). The rectal temperature was controlled at $37 \pm 0.5^{\circ} \mathrm{C}$ by a blanket heater. Antibiotics were administrated topically and systemically for 1 week to protect against infection. Two weeks after surgery, the monkey was retrained. Performance criterion was again attained in about 10 days. All experimental protocols were performed in accordance with the guidelines for care and use of laboratory animals approved by the University of Toyama and the National Institutes of Health's Guide for the Care and Use of Laboratory Animals, and approved by the Committee for Animal Experiments at the Toyama Medical and Pharmaceutical University (University of Toyama).

\section{RECORDING PROCEDURES AND DATA ACQUISITION}

After recovery from training and surgery, extracellular unit activities were recorded from the $\mathrm{HF}$ and $\mathrm{PH}$ using a tungsten electrode while the head of the monkeys were painlessly fixed in the stereotaxic apparatus using the surgically implanted U-shaped aluminum plate (see Matsumura et al., 1999). A glass-insulated tungsten microelectrode (1-2 M $\Omega$ at $1000 \mathrm{~Hz}$ ) was stereotaxically inserted vertically into the HF and $\mathrm{PH}$ stepwise by a pulse motor-driven manipulator (SM-21; Narishige, Tokyo, Japan). The diameter of the tungsten electrodes was less than $5 \mu \mathrm{m}$. The mean firing rates of the $\mathrm{HF}$ and $\mathrm{PH}$ putative pyramidal neurons (place 
cells) were less than 1 spikes/s (Matsumura et al., 1999), which are comparable to those in the monkey HF by Skaggs et al. (2007) using tetrodes. Outputs from the amplifier were digitized and sent to an IBM-compatible 486-based microcomputer. The software (Enhanced Discovery, DataWave Technologies) collected an epoch of the digitized analog signals for every event which exceeded a user-set threshold. The digital outputs of X and Y coordinates of the cab and/or pointer were simultaneously displayed on-line on another monitor (PC-9821, NEC). Each task consisted of 12 trials which contained 12 different combinations of starting points and target areas ( 4 starting points $\times 3$ target areas). Eye movements were also monitored by an eye monitor system using an infrared chargecoupled device (CCD) camera (EM100, Toyo Sangyo Co., Ltd.).

\section{SINGLE UNIT ISOLATION}

One to four single units were isolated by means of off-line cluster analysis from a single channel. Single unit isolation was performed manually using eight waveform parameters (positive, negative, and entire spike amplitudes, spike duration, amplitude windows immediately prior to and after the initial negative-going peak, and time until maximum of positive and negative peaks) with the DataWave software. First, units were isolated using parameters of entire spike amplitude and spike duration. Then, clusters of the isolated units were further analyzed using other pairs of these eight parameters. Finally, superimposed waveforms of the isolated units were drawn to check invariability of the waveforms. Invariability of the waveforms was also checked during the recording sessions; data were discarded if they were not identical in early and late recording period. Superimposed waveforms were checked so that no overcut and overlap was performed for the single unit isolation. We further examined autocorrelograms (ACRs; bin, $1 \mathrm{~ms}$; bin width, $50 \mathrm{~ms}$ ) for neurons to verify that the refractory period was greater than 1-2 ms.

\section{CLASSIFICATION OF ISOLATED UNITS}

There have been no reports indicating electrophysiological characteristics of pyramidal neurons and interneurons in the HF of awake monkeys. Therefore, the isolated units were classified based on the rodent criteria: pyramidal neurons and interneurons were identified based on their firing rates and spike widths (Fox and Ranck, 1981; Mizumori et al., 1990; Jung et al., 1994). The data matrix of the firing rates and spike widths was analyzed by the cluster analysis (SPSS version 10.0.7J, SPSS Inc.) utilizing Pearson's productmoment correlation coefficients and the average linkage method (Bieber and Smith, 1986; Nishijo and Norgren, 1990, 1991). The results indicated four clusters, and these four clusters were further grouped into two clusters, i.e., pyramidal neurons vs. interneurons based on neurophysiological characteristics (Csicsvari et al., 1998, 1999; Wiebe and Stäubli, 2001; see Figure 2 in the results). The mean firing rate of each isolated single unit (spike) was computed from whole data across the four tasks ( 12 trials $\times 4$ tasks). The duration of spikes were measured at $25 \%$ of maximum spike amplitude (Csicsvari et al., 1999).

\section{FIRING RATE MAPS AND PLACE FIELDS}

In order to visualize firing patterns with respect to location of the monkey in the experimental field or the pointer on the LCD monitor, firing rate maps were created (Matsumura et al., 1999). Movable

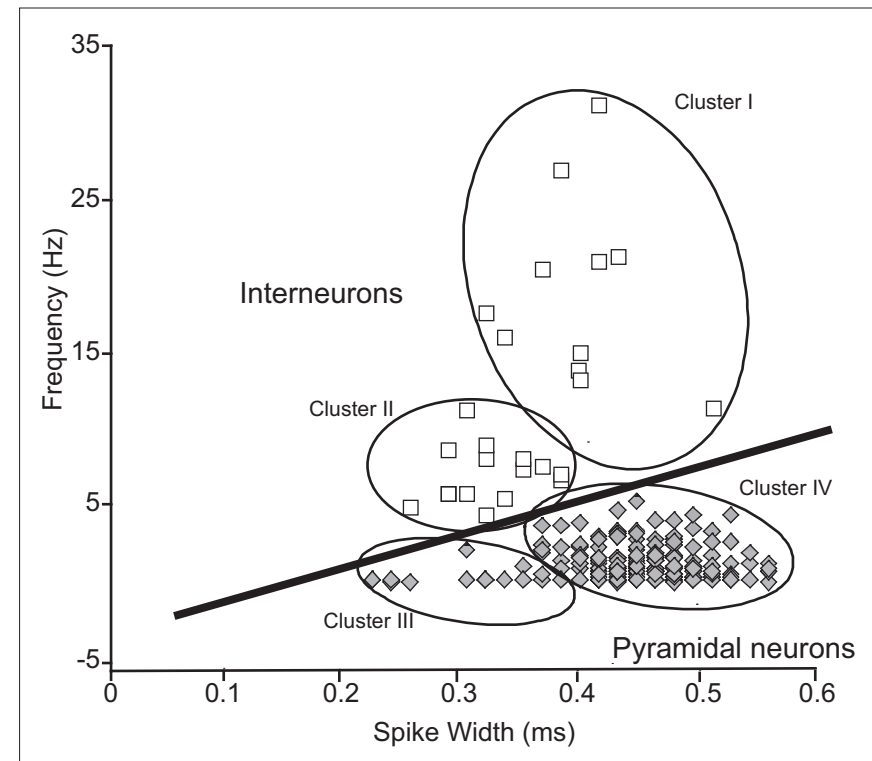

FIGURE 2 | Classification of HF neurons on the basis of physiological parameters in extracellular neuronal recordings. A linear cut-off method in firing rate-spike width space (Wiebe and Stäubli, 2001) to classify of pyramidal neurons and interneurons. HF neurons were initially grouped into the four clusters (I-IV) according to the cluster analysis. These four clusters were further grouped into two clusters (pyramidal neurons and interneurons) by the linear cut-off method. The population of pyramidal neurons was statistically well separated in the two-dimensional space $[F(2,417)=357, P<0.0001$ by two-way ANOVA]. Note that the distribution pattern and characteristics of waveforms of the pyramidal neuron and interneurons are comparable to those in rats (Jung et al., 1994; Csicsvari et al., 1999; Wiebe and Stäubli, 2001).

area in the experimental field $(2.0 \mathrm{~m} \times 2.0 \mathrm{~m})$ and that on the LCD monitor $(15.9 \mathrm{~cm} \times 15.9 \mathrm{~cm})$ were divided into 64 pixels by an $8 \times 8$ array. The mean firing rate for each pixel was calculated as the average spikes per s for all visits to that pixel during the manipulating/ response and reward phases. Then, a grand mean firing rate $(M)$ was calculated by averaging the mean firing rate across all pixels. Neuronal activity in each pixel was expressed as a relative firing rate $(R)$ in which the mean firing rate in each pixel was divided by the grand mean in each task, and shown in five steps $(R \geq 2 M$; $2.0 M>R \geq 1.5 M ; 1.5 M>R \geq 1.0 M ; 1.0 M>R \geq 0.5 M ; R<0.5 M)$.

To determine the boundary of the place field of the HF and $\mathrm{PH}$ neurons where the activity increased, we compared firing rate of each pixel with a mean firing rate over a given task (Matsumura et al., 1999). An increase in firing rates in each pixel was defined as that greater than 2.0 times the mean firing rate for a given neuron in each task. This criterion was similar to those in the previous studies (Muller et al., 1987; Kobayashi et al., 1997). Clusters of the pixels with firing rates exceeding both 1.5 and 2.0 times of the mean firing rate were identified. All pixels that did not satisfy this criterion were eliminated. Then, only place fields that had at least 1 pixel with a firing rate exceeding 2.0 times the mean and 1 adjacent pixel with a firing rate exceeding 1.5 times the mean were identified. Finally, the place fields, within which all firing rates of the pixels were less than 2.0 spikes/s (Muller et al., 1987; Breese et al., 1989), were excluded. The remained place fields could be expanded through any edge shared by 2 pixels meeting the criterion (greater 
than 1.5 times the grand mean). If one or more neighboring pixels satisfied the criterion, the field was expanded to include the pixel(s). Each added pixel was then tested for the presence of a neighboring pixel that met the criterion. When no neighboring pixel satisfied the criterion, the limit of the field was identified. Boundaries of a place field were established by constructing a rectangle that had one diagonal connecting the minimum $\mathrm{X}$ and $\mathrm{Y}$ coordinates with the maximum $\mathrm{X}$ and $\mathrm{Y}$ coordinates.

\section{CCR AND ACR ANALYSIS}

Shift register difference CCRs with a $5 \mathrm{~ms}$ bin width and a $\pm 300 \mathrm{~ms}$ binning range were employed over 12 trials in each task. Each observation time comprised about $150 \mathrm{~s}$ period including a trial and an inter-trial interval. To make a shift register difference CCR, a raw CCR was first calculated by counting the discharge numbers of target cells at every discharge of reference cells in each $5 \mathrm{~ms}$ bin over $\pm 300 \mathrm{~ms}$. Second, a shift register CCR was computed between discharges of a reference cell synchronized with an event trigger (start trigger of each trial) and discharges of a target cell synchronized with a subsequent event trigger. Then, the latter CCR was subtracted from the former CCR, which could result in negative peaks. This shift register difference CCR can prevent detection of pseudo positive peaks when two cells have a same stimulus dependence and behavioral correlate (Aertsen et al., 1989; Munk et al., 1995; Nowak et al., 1995). Finally, spike counts in each bin of the CCR histograms were normalized by division of a sum of the reference and target cell discharges according to the method by Hirase et al. (2001). The criterion for statistical significance was considered to be $P<0.001$ of confidence limits according to the methods by Perkel et al. (1967) and Gochin et al. (1989). Confidence limits are based on the assumption that, for independently firing spike trains, counts in every bins of crosscorrelogram (CCG) are modeled as a Poisson process (Perkel et al., 1967; Gochin et al., 1989). DataWave system proprietary programs were used for CCR analyses.

Additional CCR analyses were further performed with a $1 \mathrm{~ms}$ bin width and $\mathrm{a} \pm 30 \mathrm{~ms}$ binning range when the time difference of significant highest peaks were close to $0 \mathrm{~ms}$, to analyze a precise distribution of the highest peaks (see examples in Figures 4Ad and $5 \mathrm{Ab})$. Data of time differences from -1 to $+1 \mathrm{~ms}$ was discarded due to the limit of temporal precision from a single electrode.

Task-dependency of CCRs is defined as such when shift register difference CCRs showed significance in at least one but not all four tasks. We also defined that neuronal pairs are without peak shift, when their CCG peaks were centered at 0 ms (CCG peaks were generally symmetrical). In some neuron pairs with symmetrical CCG peaks centered at about time zero, their peak points could not be discerned precisely because of broad peaks. They were included in a group without a peak shift. Also, discharge patterns of each of neuron pairs were analyzed with ACRs. Spike counts in each bin of the ACR histograms were normalized by division of a sum of the discharges of the neuron.

\section{RESULTS \\ IDENTIFICATION OF PYRAMIDAL NEURONS AND INTERNEURONS BY EXTRACELLULAR RECORDINGS}

Distributions of the monkey HF neurons in firing rate-spike width space are shown in Figure 2. The cluster analysis indicated four clusters of the HF neurons (Clusters I-IV). The previous results in rodents reported that typical interneurons had relatively narrow duration and high firing rates $(>5 \mathrm{~Hz})$ while pyramidal neurons had wide duration and low firing rates (<5 Hz; Csicsvari et al., 1998, 1999; Wiebe and Stäubli, 2001). Based on these results, Clusters I and II, and Clusters III and IV were combined to form two new larger clusters (open squares vs. shaded diamonds). This distribution pattern was well comparable to that in rodents (Csicsvari et al., 1998, 1999; Wiebe and Stäubli, 2001); Clusters I and II, and Clusters III and IV corresponded interneurons and pyramidal neurons, respectively. Typical waveforms of pyramidal neurons and interneurons are shown in Figure 3, in which the two neuron pairs from A to $\mathrm{F}$ were recorded from the same electrodes.

\section{CHARACTERISTICS OF HF NEURONS AND TYPES OF NEURON PAIRS}

The $209 \mathrm{HF}$ and $\mathrm{PH}$ neurons (pyramidal neurons, 183; interneurons, 26) which had more than one isolated neuron partner recorded simultaneously were isolated. Of these 209 neurons, 163 neuron pairs were identified. The mean firing rate of the putative pyramidal neurons was $1.25 \pm 1.18$ spikes/s (mean \pm SD; range: $0.01-5.20$ ) and those of the putative interneurons was $12.39 \pm 7.67$ spikes/s (range: 5.45-31.09). The number of neuron pairs in each subregion of the HF and PH, classified by types of neuron pairs, is shown in Table 1. Of the 163 neuron pairs, 98 showed significant peaks in the CCR histograms. Thus, about two thirds of the neuron pairs had significant peaks in at least one CCR histogram of the four tasks. The most common type of the neuron pairs consisted of two pyramidal neurons (118 pairs), followed by pyramidal neuron and interneuron (32 pairs). The least common type was that of two interneurons (13 pairs). These results were attributed to the fact that the number of pyramidal neurons was larger than that of interneurons (about 10\% of pyramidal neurons) in the HF (Woodson et al., 1989; Aika et al., 1994).

\section{TASK-DEPENDENT CCRS WITH OR WITHOUT CHANGES OF THE FIRING RATES}

An example of a task-dependent CCG peak for a pair of CA1 pyramidal neurons (529U1 and 529U2, these waveforms are shown in Figures 3Aa,b, respectively) is shown in Figure 4A. This pair showed significantly higher CCG peak only in the VT/P-TC task, indicating that the functional connectivity between these two neurons strengthened when spatial information on the LCD monitor was required. The CCR histogram with $1 \mathrm{~ms}$ bin width (inset in Figure 4Ad) indicated that the highest peak shifted $3 \mathrm{~ms}$ from time zero. It should be noted that this positive peak was independent of the average firing rates during the overall recording session in each task since spike counts in each bin of the CCR histograms were normalized by dividing by the sum of the both reference and target cell discharges. ACRs of these neurons showed sharp peaks in 529U1 (Figure 4B) and broad peaks in 529U2 (Figure 4C). These ACRs indicate that $529 \mathrm{U} 1$ discharged sporadically in a bursting manner, and $529 \mathrm{U} 2$ discharged relatively tonically. This bursting pattern of the both neurons became more evident in the VT/P-TC task (Figures 4Bd,Cd), in which significant CCR was observed. Weak 10-14 Hz oscillatory activity was also observed in neuron 529U1.

Figure 5A shows another example of a task-dependent CCG peak, independent of the average firing rate, for a pair of CA1 pyramidal neurons (728U1 and 728U2, these waveforms are 


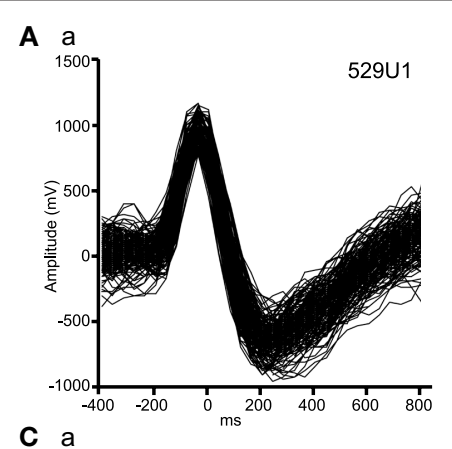

C

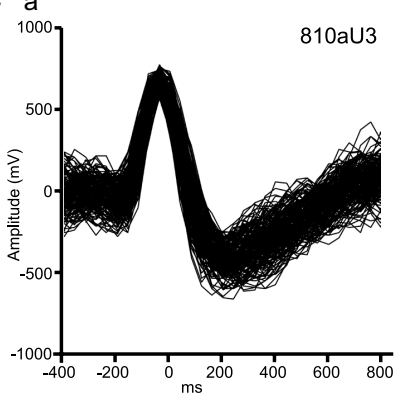

E a

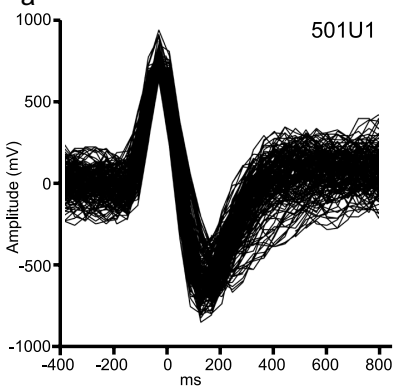

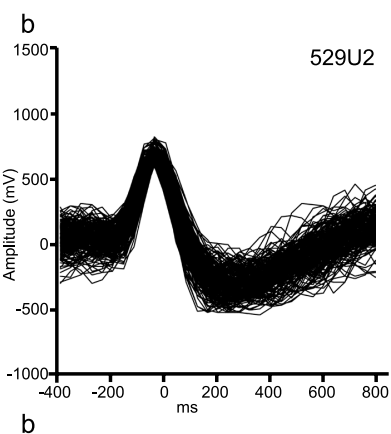
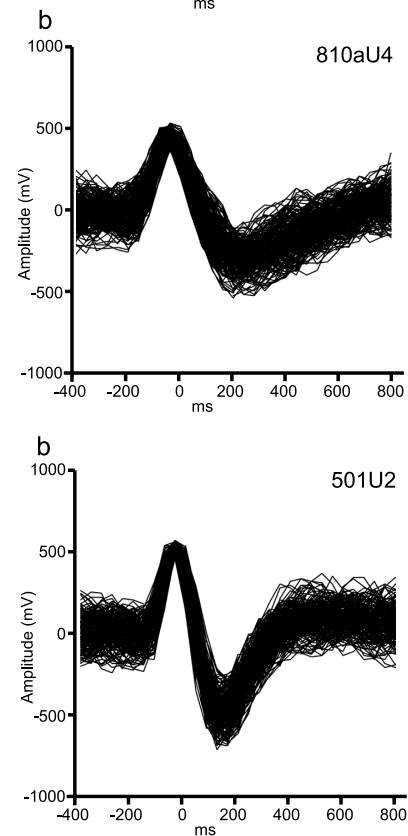

FIGURE 3 |Typical waveforms of putative pyramidal neurons ( $A a, A b, B a$, $\mathrm{Bb}, \mathrm{Ca}, \mathrm{Cb}, \mathrm{Da}, \mathrm{Ea}, \mathrm{Fa}, \mathrm{Fb})$ and an interneuron ( $\mathrm{Db}, \mathrm{Eb})$ isolated by off-line cluster analysis. The two units in a same uppercase letters were simultaneously recorded from a same electrode. The ACR and CCR of these
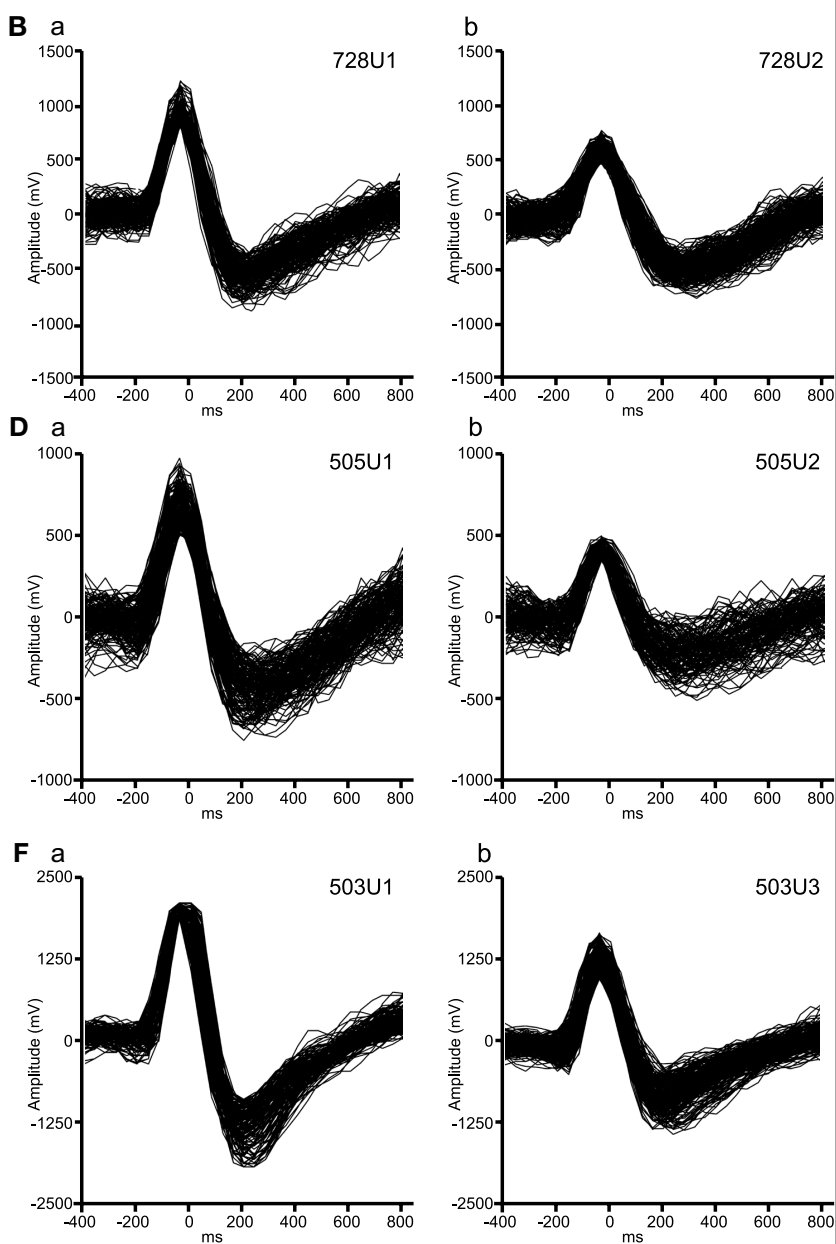

neurons were shown in Figures 4-10. The unit pairs of (A) (Figure 4) and (B)

(Figure 5) show task-dependent CCRs, whereas the unit pairs of (C) (Figure 6),

(D) (Figure 7), and (E) (Figure 8) show task-independent CCRs. The unit pair (F)

(Figure 10) shows task-dependent CCG peak with trigger-like activity. shown in Figures $3 \mathbf{B a}, \mathbf{b}$, respectively). This pair showed a significant CCG peak only in the RT/P-TC task. This suggests that the functional connectivity strengthened when both real and virtual information was required. Similarly, this pair had a peak shift in $4 \mathrm{~ms}$ (inset in Figure 5Ab). ACRs of these neurons (Figures 5B,C) showed generally similar patterns to those of the neuron pair in Figure 4. Although $728 \mathrm{U} 1$ discharged in a similar bursting manner across the tasks, bursting pattern of $728 \mathrm{U} 2$ became more evident in the RT/P-TC task (Figure 5Cb) in which significant CCR was observed.

Thus, the neuron pairs with task-dependent CCG peaks had some common characteristics. First, most pairs were comprised of pyramidal vs. pyramidal neurons. Especially, when the significant CCG peaks were observed only in one task, all the pairs were pyramidal vs. pyramidal neurons. Second, almost all CCG peaks were positive. Especially, all pairs had positive peaks when the significant CCR was selective only to one task. Third, CCG peaks of most pairs shifted with time differences less than $50 \mathrm{~ms}$ (see also Figure 13).

\section{TASK-INDEPENDENT CCRS}

Figure 6A shows a typical example of positive peaks in CCR histograms ( $\pm 300 \mathrm{~ms}$ binning range, $5 \mathrm{~ms}$ bin width), between a pair of CA1 pyramidal neurons (810aU3 and $810 \mathrm{aU} 4)$, which occurred non-differentially in the four tasks. Significant positive peaks, which centered at about time zero with symmetrically broad bases between about $\pm 200 \mathrm{~ms}$, were observed in all four CCR histograms. These data indicated that the two neurons discharged mostly at same time, and synchrony of the two neurons was task-independent. This CCR pattern was frequently observed in the combinations of pyramidal vs. pyramidal neurons and of pyramidal neuron vs. interneuron. Their peaks occurred at about time zero (no clear peak shift) in half of the pairs of pyramidal neuron vs. interneuron, while most pairs of pyramidal vs. pyramidal neurons displayed a peak shift. ACRs ( $\pm 300 \mathrm{~ms}$ binning range and $5 \mathrm{~ms}$ bin width) of these neurons are shown in Figure 6B. Both neurons 810aU3 (Figure 6Ba) and 810aU4 (Figure 6Bb) showed a bell-shaped ACR. This suggests that these neurons discharged in a similar bursting rhythm. 
A CCG 52901 vs. $529 U 2$
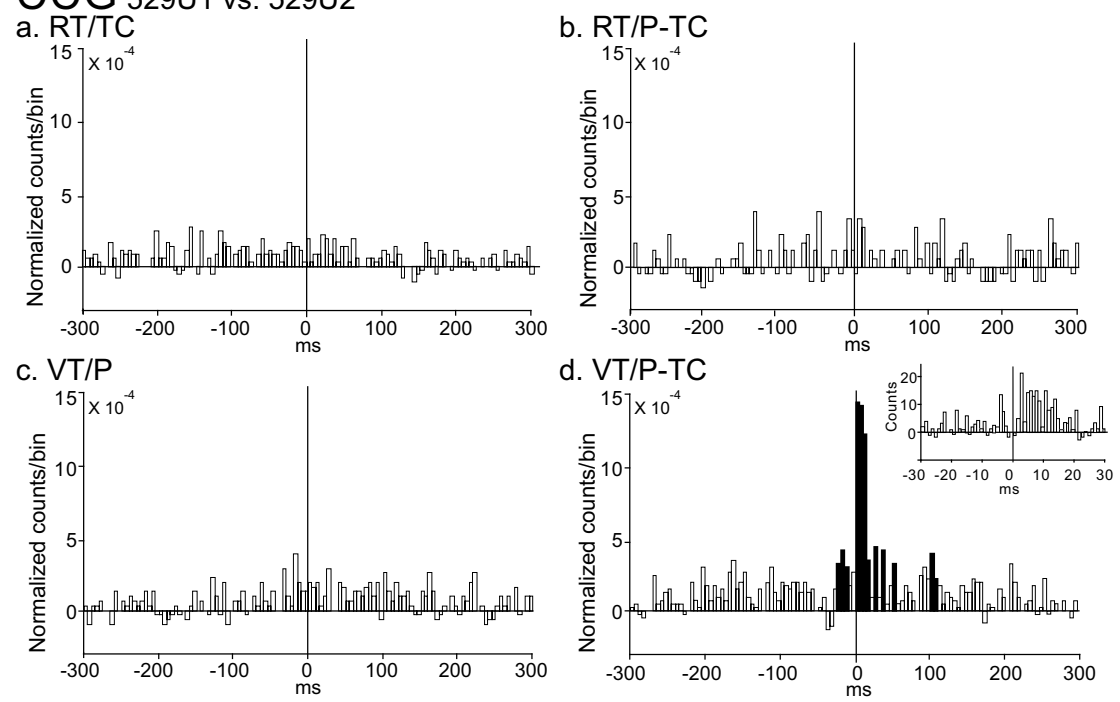

B ACR 529U1

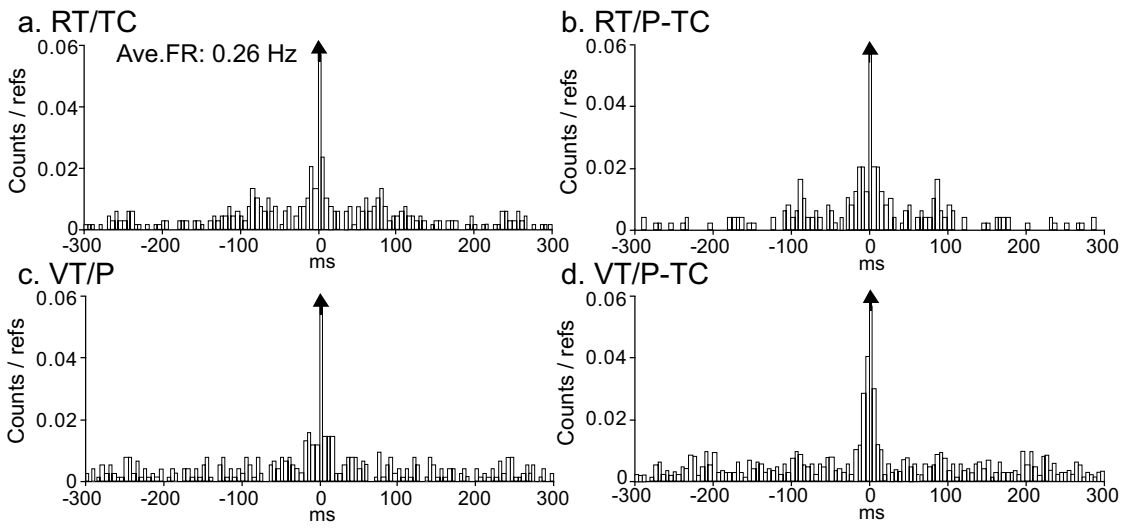

c ACR 52902

a. RT/TC
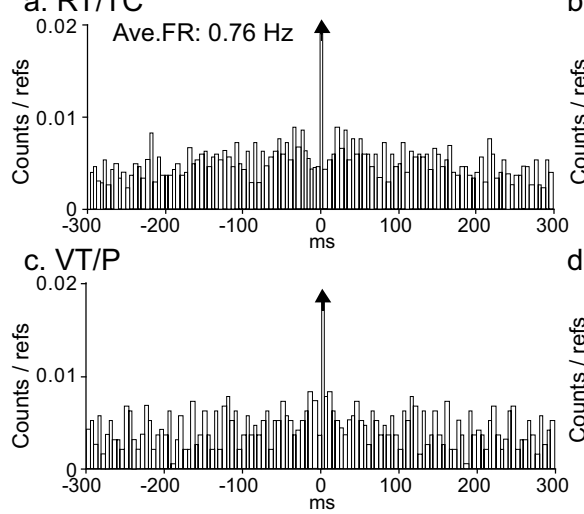

b. RT/P-TC

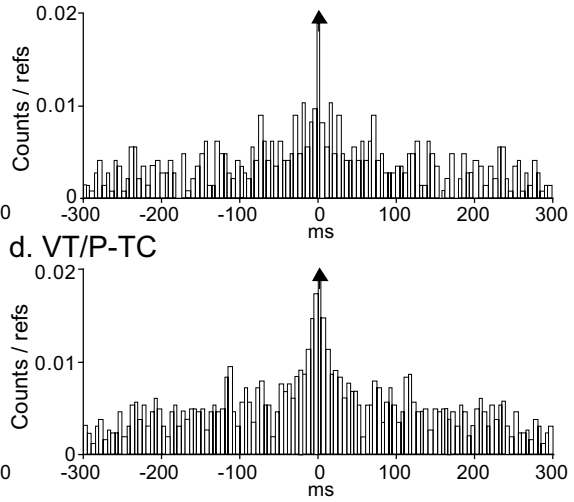

FIGURE 4 |An example of a neuron pair displaying a task-dependent peak in the CCGs. (A) Difference CCGs for a pair of CA1 pyramidal neurons (529U1, reference cell; 529U2, target cell) in the four tasks (a-d). Note a significant peak in the VT/TC-P task. (d). Inset in (d), CCR histogram with $1 \mathrm{~ms}$ bin width. Total spikes analyzed in each CCG in each task for neurons 529 U1 and 529 U2 were 382 and 1419 spikes in the RT/TC, 203 and 606 spikes in the RT/P-TC, 333 and 745 spikes in the VT/P, and 282 and 609 spikes in the VT/P-TC tasks, respectively. Hatched bins indicate those exceeding $99.9 \%$ of confidence limits. Ordinate, spike counts of the referenced cell (spike counts in each bin of the CCR histograms were divided by both sums of the reference and target cell discharges). Abscissa; time difference from each spike of the reference cell to that of the target cell. (B,C) ACRs of the same neurons in (A) [(B) 529U1; (C) 529U2]. Note a sharp peak in 529U1, and a broad peak in 529U2. It is noted that $529 U 1$ and 529 U 2 were not complex spike cells. Ave. FR, average frequency of a neuron across the four tasks. Ordinate, spike counts of the cell. Abscissa; time difference from each reference spike to each spike of the cell. 


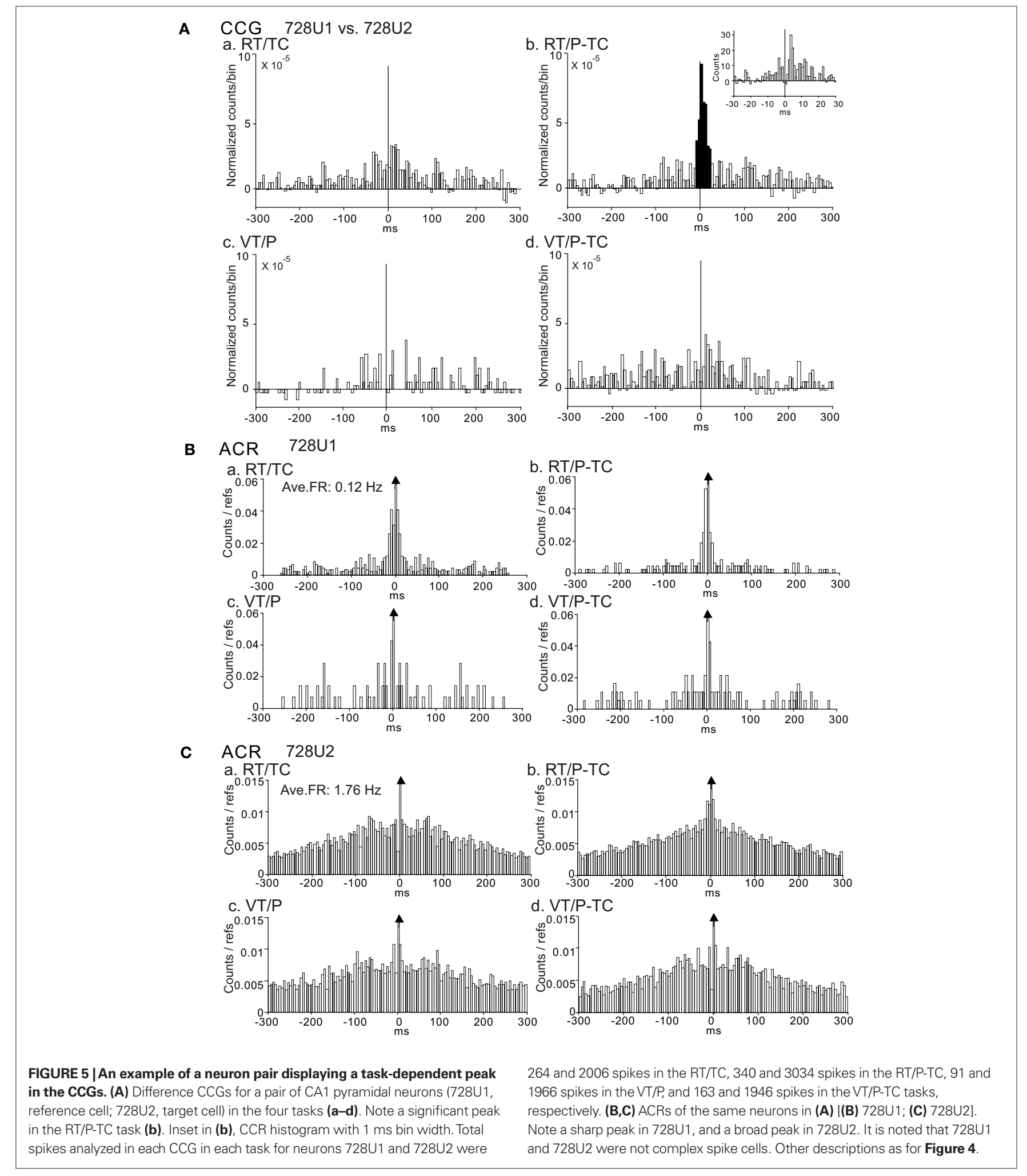

A typical example of task-independent negative CCG peaks between a pair of CA1 pyramidal neuron (505U1: reference neuron) and interneuron (505U2: target neuron) is shown in Figure 7. Significant negative peaks centered at about time zero, with sym- metrically broad bases ranging from -35 to $+35 \mathrm{~ms}$ latency, were observed in all CCR histograms in the four tasks (Figure 7A). These results indicate that the synchrony of these neurons is task-independent. Patterns of ACRs in these neurons (505U1 and 505U2) 


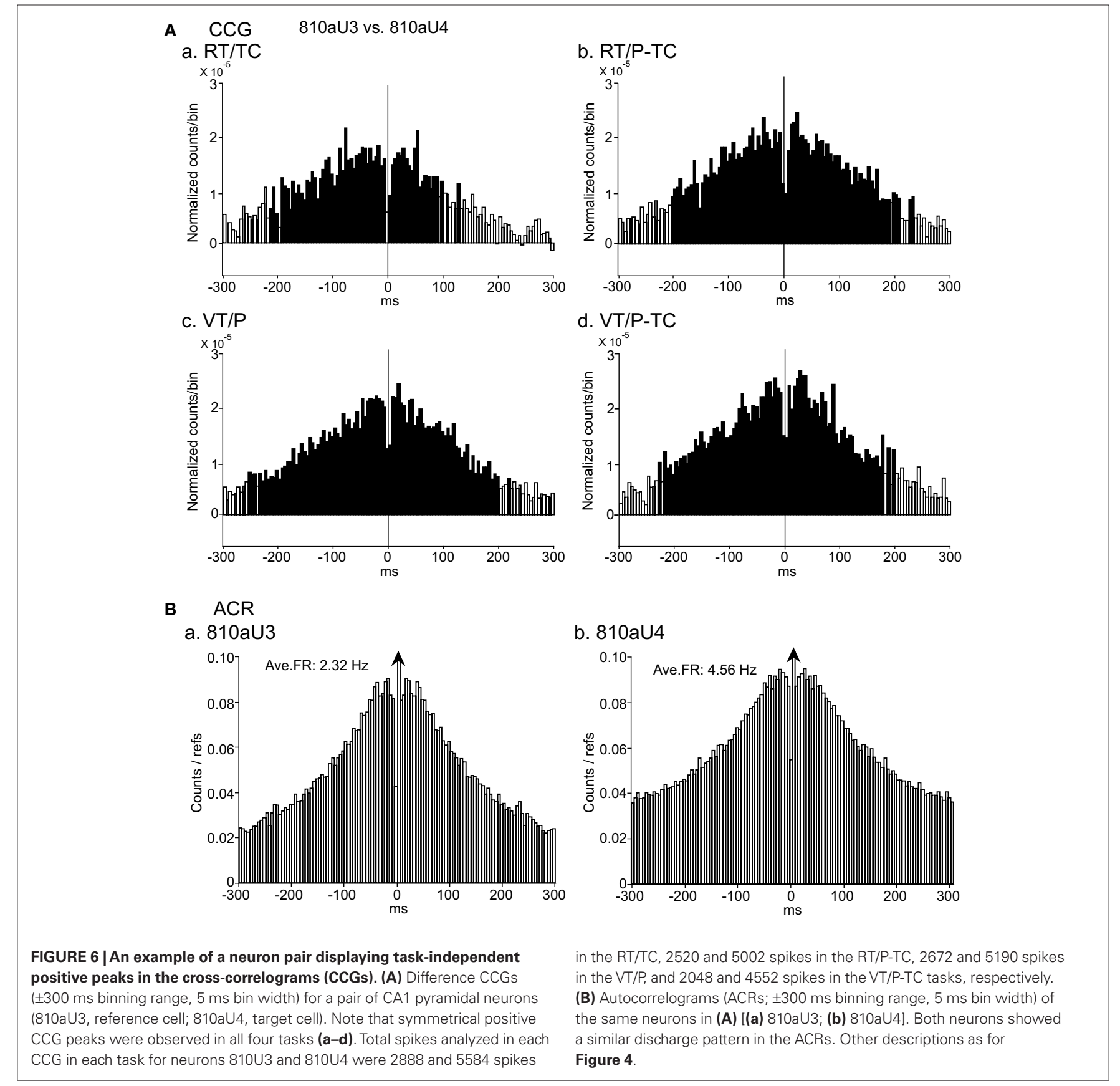

were similar; inhibition around their discharges with long duration (about $\pm 50 \mathrm{~ms}$; Figure 7B). Four of five pairs with the negative CCG peaks, which were observed in all three types of neuron pairs, displayed task-independent correlation without a peak shift.

Figure 8A shows a typical example of task-independent centernegative and surround-positive CCG peaks between a pair of CA1 pyramidal neuron (501U1, reference cell) and interneuron (501U2, target cell). Both center-negative and surround-positive peaks were symmetrical at time zero and task-independent. The significant negative peaks were narrower than those in the pair of Figure 7A (about $\pm 10 \mathrm{~ms}$ ), and the significant positive peaks had broad bases ranging from -250 to $+250 \mathrm{~ms}$. The patterns of ACRs of these neurons (501U1 and 501U2) were similar (Figure 8B); inhibition around their discharges (i.e., time zero in the ACR histograms) lasted for a relatively short duration (about $\pm 20 \mathrm{~ms}$ ). Eleven of 14 pairs with the center-negative and surround-positive CCG peaks, which were comprised of all three types of neuron pairs, showed task-independent correlations, and 13 of these 14 pairs had no peak shift in the CCR histograms.

\section{THREE HZ OSCILLATION IN CCGS}

A few oscillatory activities were observed in the CCR histograms (3/98 pairs). An example of an oscillatory CCG peak between a pair of CA1 pyramidal neurons (811U1 and $811 \mathrm{U} 2)$ in RT/TC task is 
A CCG $505 \mathrm{U} 1$ vs. $505 \mathrm{U} 2$

a. RT/TC

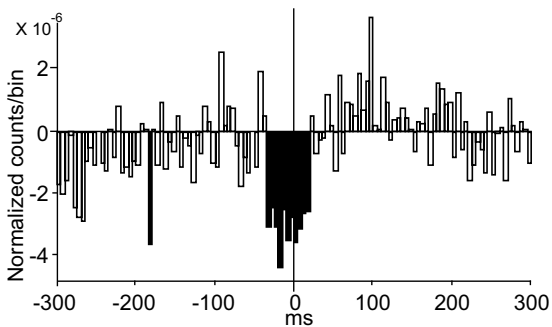

c. VT/P

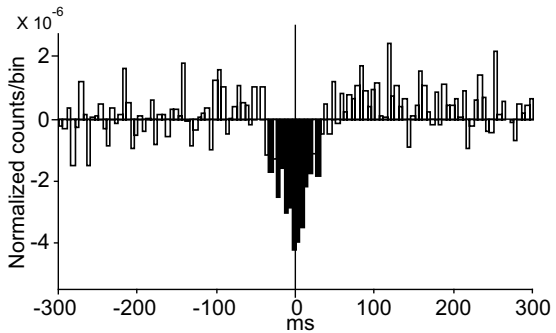

B $\quad$ ACR

a. $505 \mathrm{U} 1$

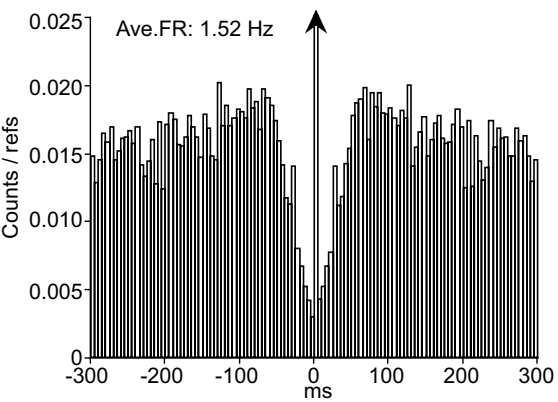

FIGURE 7 |An example of a neuron pair displaying task-independent negative peaks in the CCGs. (A) Difference CCGs for a pair of CA1 pyramidal neurons (505U1, reference cell; 505U2, target cell) in the four tasks (waveforms are shown in Figures $\mathbf{3} \mathbf{D a}, \mathbf{b})$. Note that broad symmetrical negative peaks were observed in the four tasks (a-d). Total spikes analyzed in each CCG in each task

shown in Figure 9A. It is noted that this pair displayed the positive CCG peak with a peak shift and this CCG peak was task-dependent. This task-selectivity was dependent on the firing rates of both neurons $811 \mathrm{U} 1$ and $811 \mathrm{U} 2$ (data not shown). ACR analysis indicated clear $3 \mathrm{~Hz}$ oscillation in $811 \mathrm{U} 1$ (Figure 9Ba), but not in $811 \mathrm{U} 2$ (Figure 9Bb). Thus, the oscillation observed in the CCR histogram of this pair was attributed to oscillatory activity of the neuron 811U1. All three pairs with the oscillatory CCG peaks displayed $3 \mathrm{~Hz}$ oscillation, and were comprised of pyramidal neuron vs. pyramidal neurons. Two of these three pairs had task-independent CCG peaks and the remaining one had the task-dependent CCG peak.

\section{TRIGGER-LIKE NEURONAL DISCHARGES WITH TASK-DEPENDENCY}

Three neuron pairs displayed interesting CCR histograms, in which one neuronal discharge (503U1) preceded activity of another neuron (503U3; Figure 10A, waveforms are shown in

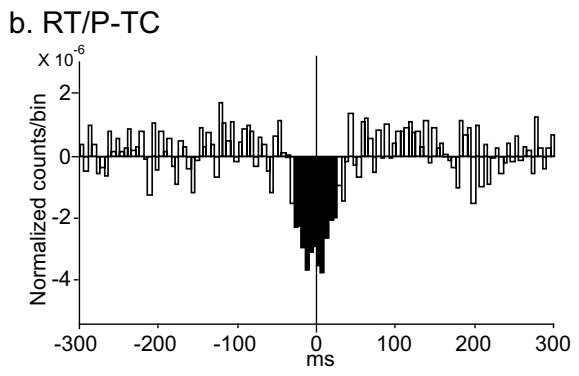

d. VT/P-TC
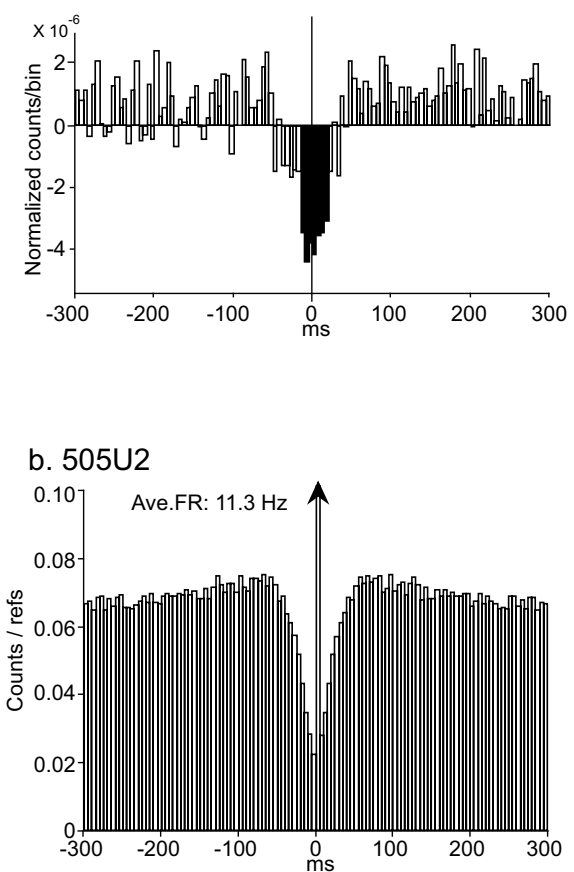

for neurons 505U1 and 505U2 were 2873 and 13900 spikes in the RT/TC, 1204 and 10187 spikes in the RT/P-TC, 1524 and 14661 spikes in the VT/P, and 1145 and 8365 spikes in the VT/P-TC tasks, respectively. (B) ACRs of the same neurons in (A) [(a) 505U1; (b) 505U2]. Other descriptions as for Figure 4.

Figure 3F). Significant positive CCG peaks between the CA1 pyramidal neurons occurred $5 \mathrm{~ms}$ after time zero and lasted for more than $300 \mathrm{~ms}$. It is noted that the positive CCG peak was task-selective and dependent on the average firing rate of both neurons 503U1 and 503U3 (data not shown). ACRs of neurons 503U1 (Figure 10Ba) and 503U3 (Figure 10Bb) showed bellshaped peaks, but the peak was steeper in 503U1 neuron. All of these three pairs showed same characteristics, i.e., combination of pyramidal vs. pyramidal neurons, the firing rate-dependent task-selectivity, and peak shift.

\section{RELATIONSHIP BETWEEN CCG PEAKS AND SPATIAL CORRELATES}

To analyze the relationships between the CCG peaks and spatial correlates of the HF neurons, firing rate maps (Matsumura et al., 1999) were created. Figure 11 illustrates the firing rate maps of a pair of 529U1 and 529U2 neurons, which showed 
A CCG $501 \mathrm{U} 1$ vs. 501U2

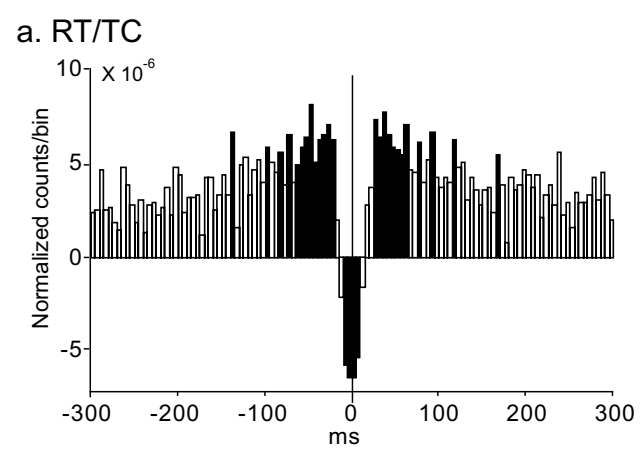

c. VT/P

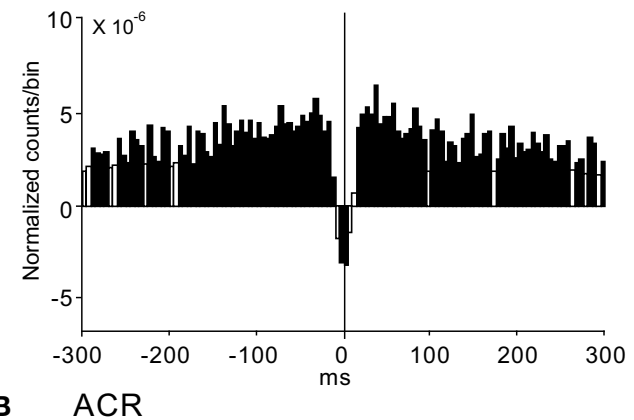

B ACR

a. $501 \mathrm{U} 1$

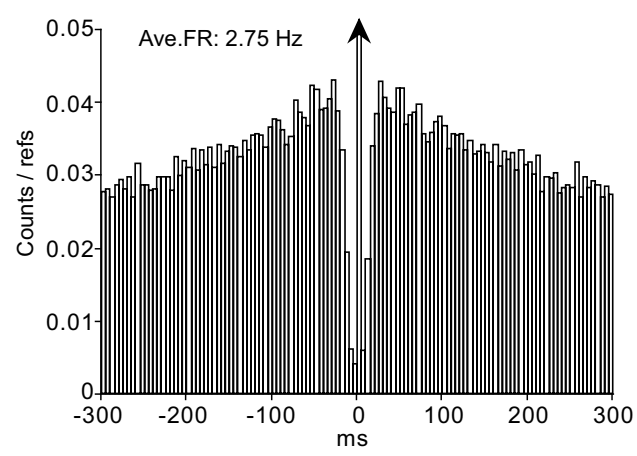

FIGURE 8 | An example of a neuron pair displaying task-independent center-negative and surround-positive peaks in the CCGs. (A) Difference

CCGs for a pair of CA1 pyramidal neuron (501U1, reference cell) vs. interneuron (501U2, target cell) in the four tasks (a-d). Total spikes analyzed in each CCR in b. RT/P-TC

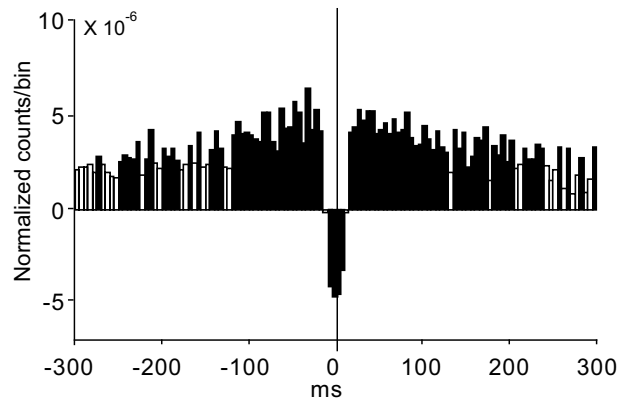

d. VT/P-TC

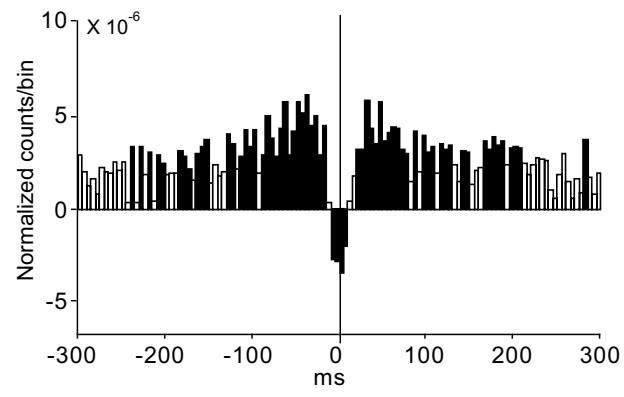

b. $501 \mathrm{U} 2$

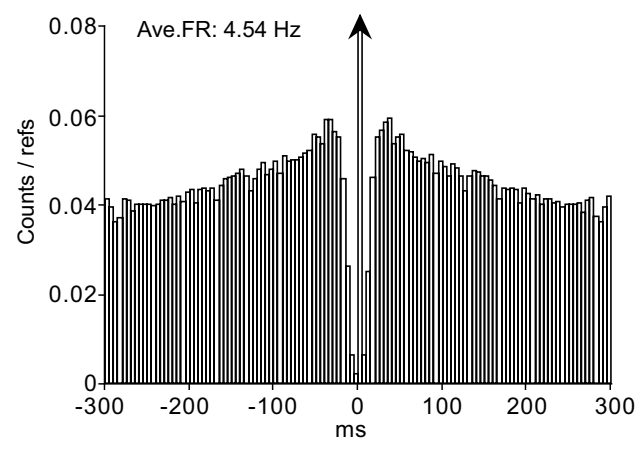

each task for neurons $501 \mathrm{U} 1$ and $501 \mathrm{U} 2$ were 1714 and 6623 spikes in the RT/ TC, 3763 and 5698 spikes in the RT/P-TC, 3027 and 6273 spikes in the VT/P, and 1062 and 2659 spikes in the VT/P-TC tasks, respectively. (B) ACRs of the same neurons in (A) [(a) 501U1; (b) 501U2). Other descriptions as for Figure 4 task-dependent CCRs in the VT/P-TC task (Figure 4). Neuron $529 \mathrm{U} 1$ displayed a place field only in the VT/P-TC task while neuron 529U2 displayed place fields in the RT/TC, RT/P-TC, and VT/P-TC tasks. It is noted that activity of the both neurons increased when the monkey visited around the center of the experimental field in the VT/P-TC task, so the place fields of these neurons overlapped only in the VT/P-TC task. When data sample was confined to place-differential neurons with the place fields, most neuron pairs with significant CCRs displayed overlap of the place fields in those tasks in which significant CCRs were observed; $66.7 \%$ (20/30) of the neuron pairs with task-dependent CCRs, and 73.2\% (30/41) with task-independent CCRs displayed overlap of the place fields.

\section{POPULATION ANALYSIS OF TASK-DEPENDENT CROSS-CORRELATION}

Numbers of the neuron pairs with task-dependent (44 pairs) and independent CCRs (54 pairs) in each task were shown in Figure 12A. There was no significant difference in ratio of task-dependent and independent CCRs among four tasks $\left(\chi^{2}\right.$-test, $\left.P>0.05\right)$. This indicates that there is no apparent difference of the number of neuronal populations relating task-dependency among the tasks. Next, to test strength of coactivation of the task-dependent neuronal populations, mean normalized counts between -50 and +50 ms were compared between task-dependent significant and non-significant CCRs by two-way analysis of variance (ANOVA; task $\times$ CCR significance; Figure 12B). The results indicated that there was a significant main effect of CCR significance $[F(1,154)=31.584, P<0.0001]$, 


\section{A $\quad \mathrm{CCG}(\mathrm{RT} / \mathrm{TC}) \quad$ 811U1 vs. $811 \mathrm{U} 2$}

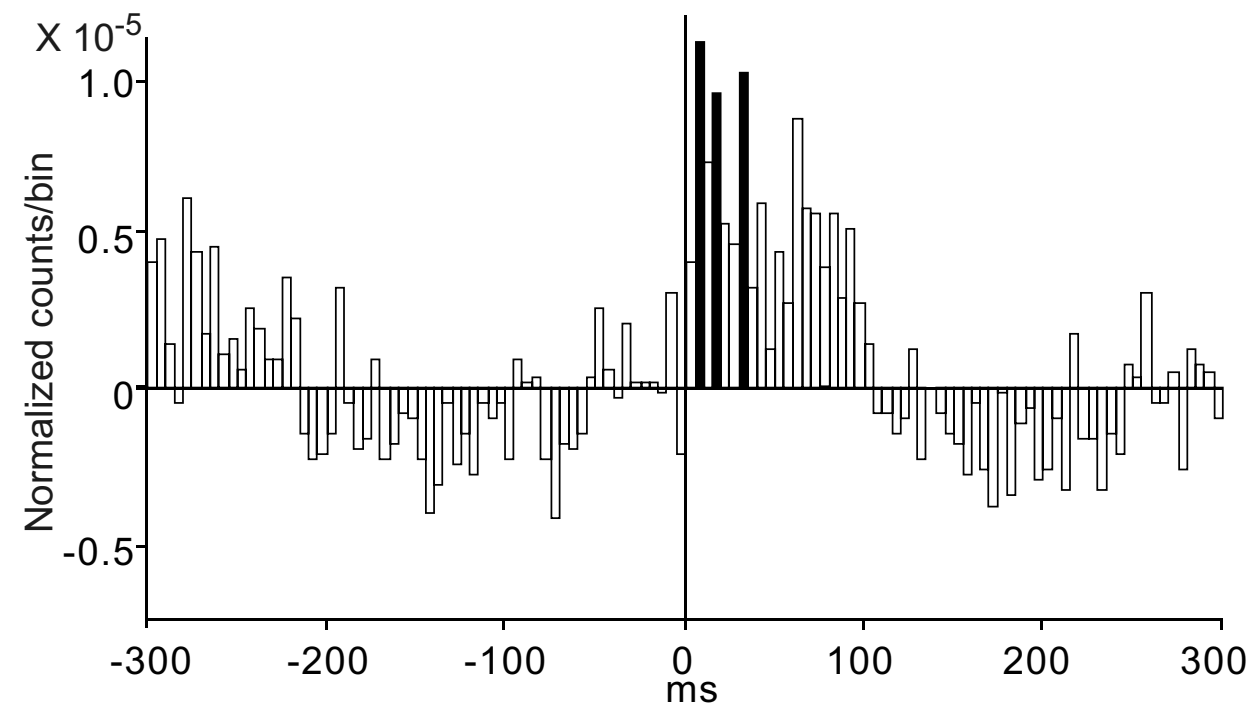

B ACR

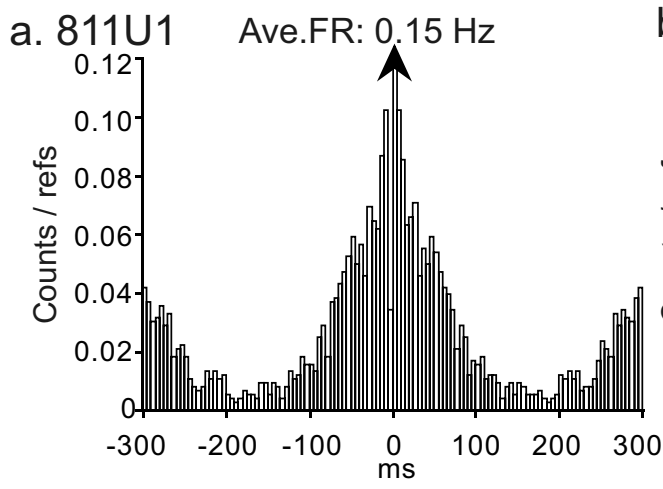

b. $811 \mathrm{U} 2$ Ave.FR: $2.54 \mathrm{~Hz}$

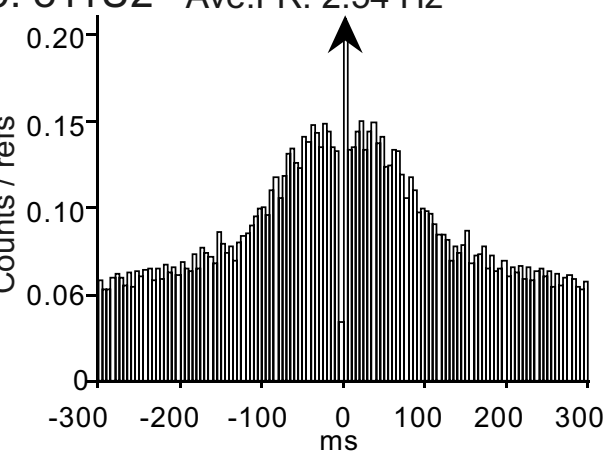

FIGURE 9 |An example of a neuron pair displaying a task-dependent peak with theta oscillation in the CCGs. (A) Difference CCGs for a pair of CA1 pyramidal neurons (811U1, reference cell; 811U2, target cell) displaying an oscillatory CCG peak in the RT/TC task. Total spikes analyzed in each CCG in each task for neurons 811 U1 and 811 U2 were 804 and 5131 spikes in the
RT/TC, 27 and 1440 spikes in the RT/P-TC, 14 and 231 spikes in the VT/P, and 14 and 374 spikes in the VT/P-TC tasks, respectively. (B) ACRs of the same neurons in (A) [(a) 811U1; (b) 811U2) indicating $3 \mathrm{~Hz}$ oscillation in $811 \cup 1$ (a), but not in 811 U2 (b). Other descriptions as for Figure 4 while there was no interaction between task and CCR significance $[F(3,154)=0.746, P>0.05]$. This indicates that specific populations of neuron pairs contribute to generation of task-dependent CCG peaks.

\section{RELATIONSHIPS AMONG TASK-DEPENDENCY, PEAK LATENCY, AND PEAK POLARITY}

Figure 13 summarizes the number of neuron pairs whose CCG peaks occurred at time difference. All peaks occurred within $50 \mathrm{~ms}$ time difference between discharges of neuron pairs, and they were observed most frequently at 0 (i.e., no peak shift) and within $10 \mathrm{~ms}$ latency.

Numbers of significant CCRs in each type of neuron pair are shown in terms of peak shift and peak polarity (left columns) in Figure 14A. There were some significant relationships among these parameters (Fisher's exact probability test, $P<0.05$ in all); (1) ratio of overall positive CCG peaks (79/163) was larger than those of negative peaks (5/163) and center-negative and surround-positive peaks (14/163), (2) ratio of positive CCG peak was larger in pyramidal vs. pyramidal neuron pairs $(67 / 163)$ than those in pyramidal neuron vs. interneuron (11/163) and interneuron vs. interneuron pairs (1/163), (3) ratio of CCG peaks with peak shift $(69 / 163)$ was larger than that of CCG peak without peak shift (29/163), (4) ratios of positive CCG peaks with peak shift were much larger in pyramidal neuron vs. pyramidal neuron pairs $(57 / 163)$ than pyramidal neuron vs. interneuron $(8 / 163)$, and none in interneuron vs. interneuron (0/163) pairs. These results indicate that pyramidal vs. pyramidal neuron pairs with positive CCG peaks and peak shift predominate in the monkey $\mathrm{HF}$ and $\mathrm{PH}$. 
A $\mathrm{CCG}(\mathrm{RT} / \mathrm{TC}) \quad 503 \mathrm{U} 1$ vs. $503 \mathrm{U} 3$

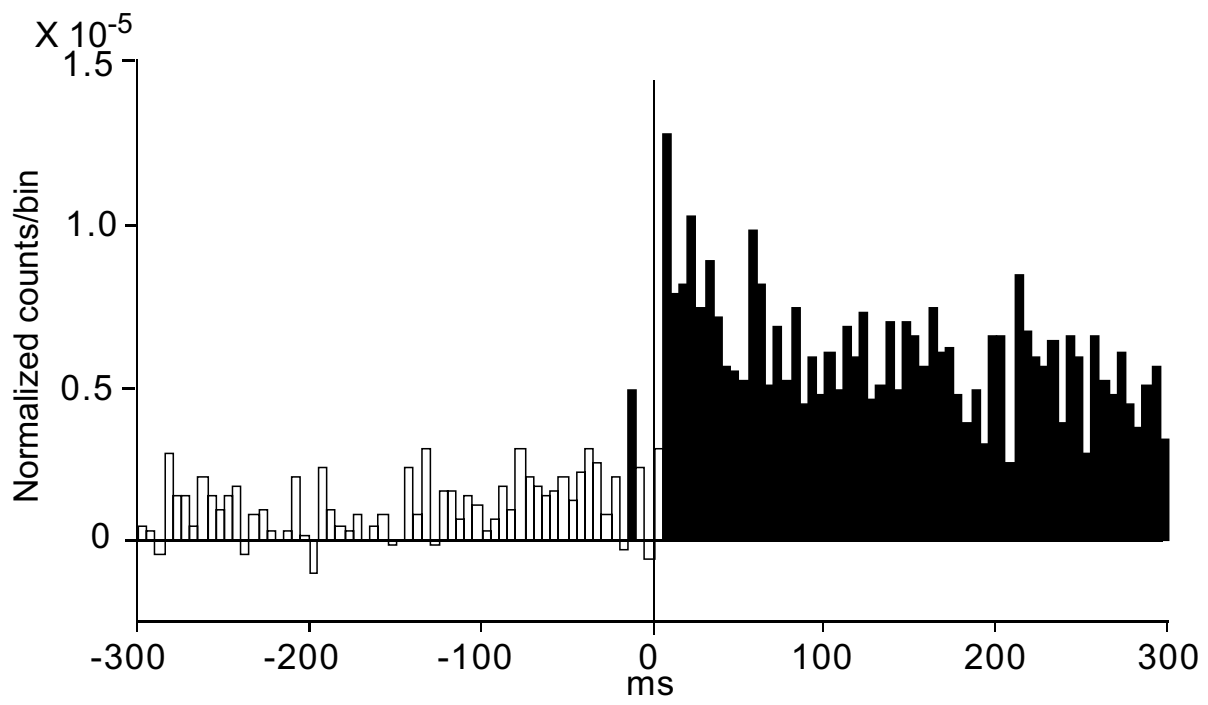

B $\mathrm{ACR}$
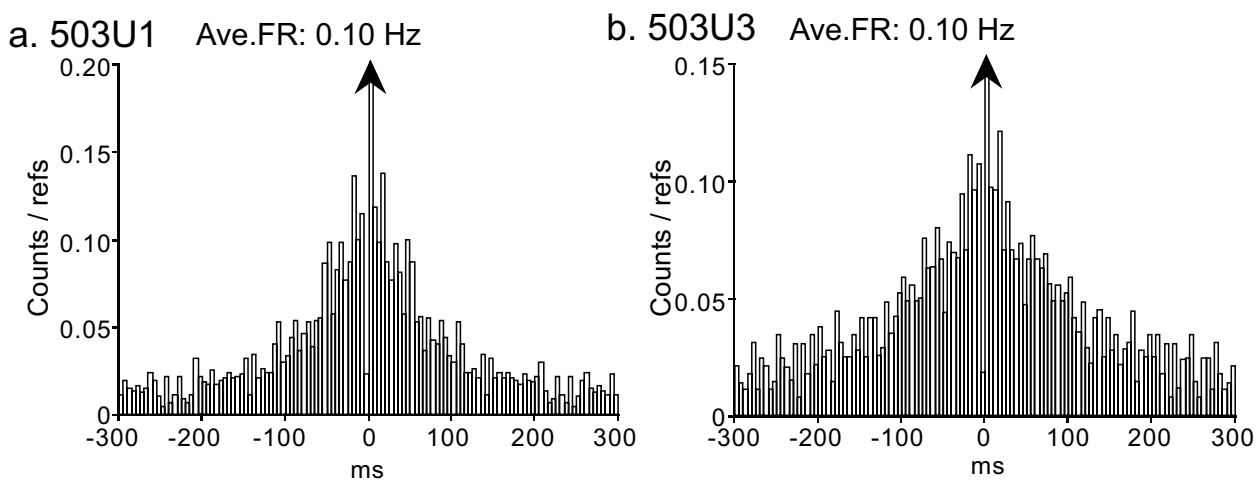

FIGURE 10 | An example of a neuron pair displaying a task-dependent peak with trigger-like activity in the CCGs. (A) Difference CCGs for a pair of CA1 pyramidal neurons (503U1, reference cell; $503 \mathrm{U} 3$, target cell) in the RT/TC task. Note that one neuronal discharges (503U1) precede activity of the other neuron (503U3). Total spikes analyzed in each CCG in each task for neurons $503 \mathrm{U} 1$ and $503 \mathrm{U} 3$ were 393 and 1837 spikes in the RT/TC, 33 and 819 spikes in the RT/P-TC, 26 and 383 spikes in the VT/P, and 78 and 1145 spikes in the VT/P-TC tasks, respectively. (B) ACRs of the same neurons in (A) [(a) 503U1; (b) 503U3]. Other descriptions as for Figure 4.
In contrast, ratio of negative CCG peaks (negative peaks and center-negative plus surround-positive peaks) were more common in the neuron pairs without peak shift $(15 / 163)$ than those with peak shift (4/163; Fisher's exact probability test, $P<0.05$; Figure 14A).

Task-dependency of significant CCG peaks in each type of neuron pairs with and without peak shift is summarized in Figure 14B. There were some significant relationships among these parameters (Fisher's exact probability test, $P<0.05$ ); (1) the task-dependent CCG peaks were found only in pyramidal vs. pyramidal neurons $(38 / 163)$ and pyramidal neuron vs. interneuron neuron $(6 / 163)$ pairs, but none in interneuron vs. interneuron pairs $(0 / 163),(2)$ ratios of task-dependent CCG peaks were more prominent in the neuron pairs with peak shift $(35 / 163)$ than the neuron pairs without peak shift $(8 / 163)$; (3) in pairs without peak shift, taskindependent CCG peaks (21/163) were more common than that of task-dependent CCG peaks (8/163), (4) task-dependent CCG peaks were more common in pyramidal vs. pyramidal neuron pairs with peak shift (30/163) than pyramidal neuron vs. interneuron $(5 / 163)$ and interneuron vs. interneuron (0/163) pairs with peak shift, (5) there were no significant differences in ratios of neuron pairs with peak shift between pyramidal vs. pyramidal neuron pairs with taskdependent (30/163) and task-independent (31/163), (6) there were no significant differences in ratios of task-independent CCG peaks between neuron pairs without (21/163) and with (34/163) peak shift. These data indicate that pyramidal vs. pyramidal neuron pairs with positive CCG peaks and peak shift contribute to both task-dependent and independent information processing, and that neuron pairs both with and without peak shift contribute to taskindependent information processing.

Overall, these results suggest some important characteristics of functional connectivity in the HF that pyramidal neuron vs. pyramidal neuron pairs with positive peak and peak shift 


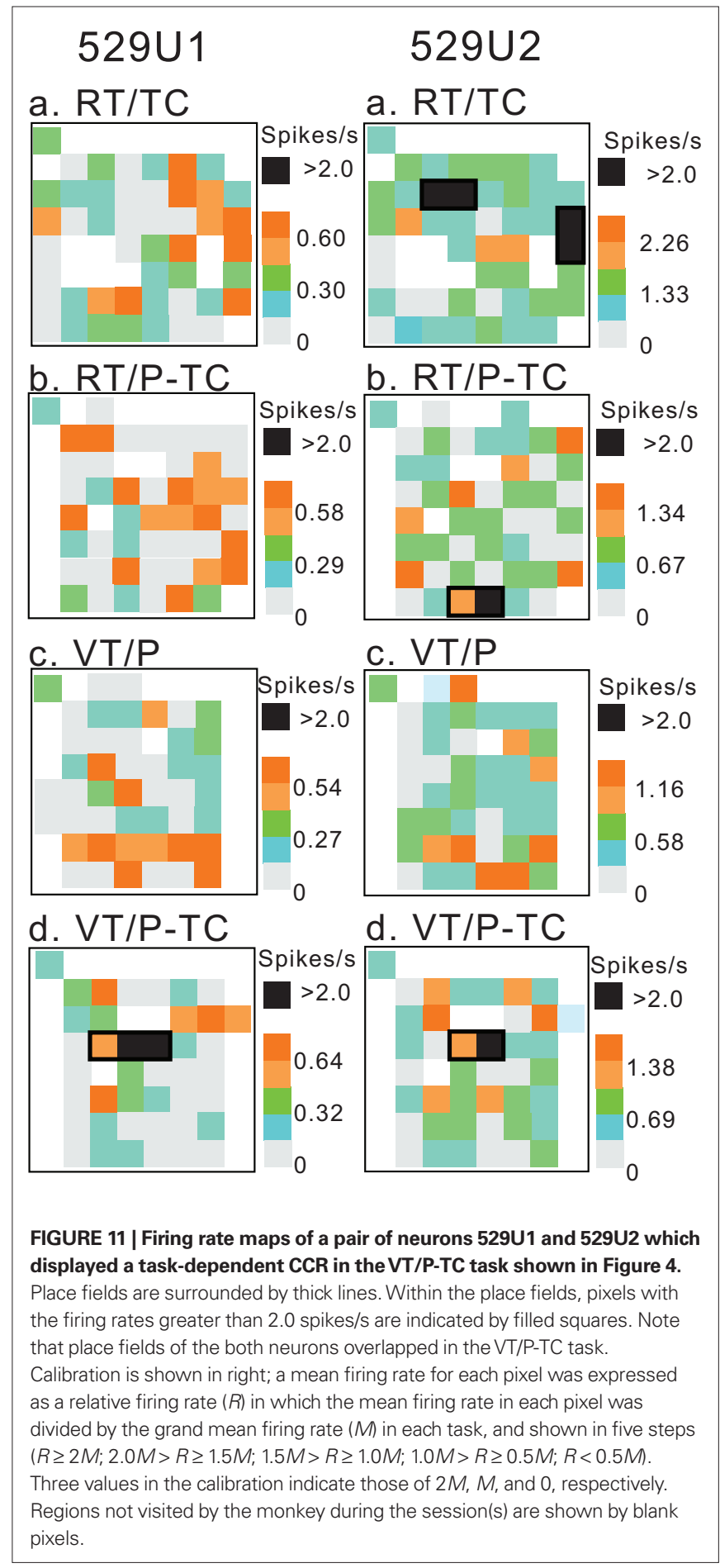

contribute to task-dependent information processing. However, no consistent patterns were observed in task-independent CCG peaks that both pyramidal neuron vs. pyramidal neuron and interneuron vs. interneuron pairs contribute to task-independent information processing, and that both neuron pairs with and without peak shift contribute to task-independent information processing.

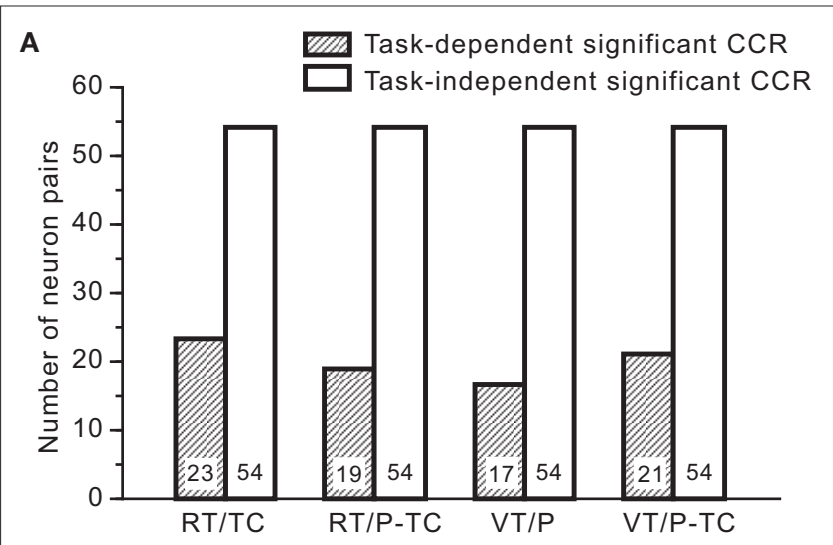

B

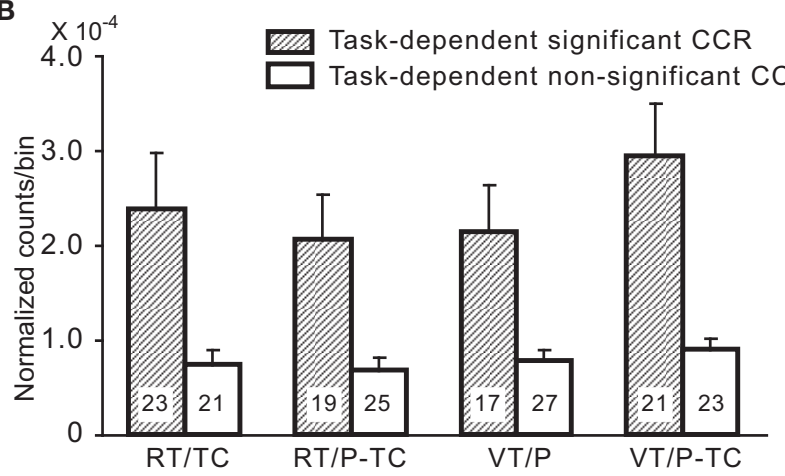

FIGURE 12 | Characteristics of the neuron pairs with the task-dependent CCG peaks. (A) Frequency histogram of task-dependent and taskindependent significant CCG peaks in each task. By definition, the same numbers of task-independent significant CCRs were found in all tasks. (B) Comparison of mean normalized counts between -50 and +50 ms in the CCRs between task-dependent significant and non-significant CCG peaks. Numbers of the neuron pairs are indicated in each column.

\section{REGIONAL DIFFERENCES AMONG SUBREGIONS IN THE HF AND PH}

Numbers of the significant and non-significant CCRs based on neuron pair types in each subregion of the HF and $\mathrm{PH}$ are shown in Table 1. Characteristics of the neuron pairs with significant CCRs in each subregion of the $\mathrm{HF}$ and $\mathrm{PH}$ regarding task-selectivity, peak shift, and peak polarity are shown in Table 2. Although most neuron pairs were recorded from the CA1 subregion and dentate gyrus, the trends of CCR characteristics in each subregion were similar to those noted in the above section; (1) pyramidal vs. pyramidal neuron pairs predominated in the all subregions, (2) neuron pairs with peak shift predominated in the all subregions, and (3) neuron pairs with positive peak polarity predominated in the all subregions.

\section{DISCUSSION}

\section{GENERAL CHARACTERISTICS}

In the present study, multiple single units were recorded from a same single electrode. Relatively high incidence of significant CCRs in the present study might be attributed to recording of neighboring neurons, consistent with previous studies (Sakurai, 1996; Takahashi and Sakurai, 2009). Strength of correlation in CCRs depended on the distance between two neurons in the auditory 


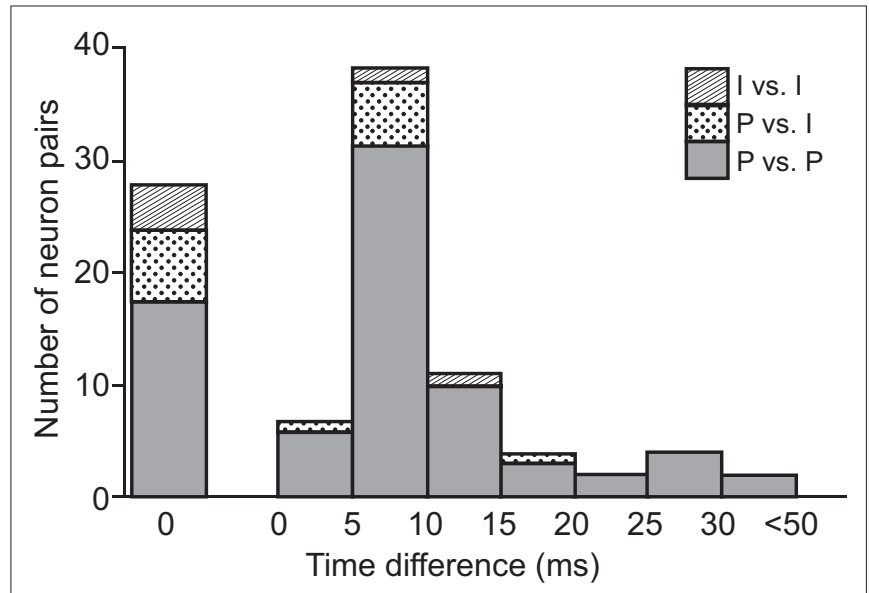

FIGURE 13 | Frequency histogram of the time differences of the CCG peaks in terms of the types of the neuron pairs. $P$ vs. $P$, pyramidal neuron vs. pyramidal neuron; P vs. I, pyramidal neuron vs. interneuron; I vs. I, interneuron vs. interneuron.

cortex (Maldonado and Gerstein, 1996). The neighboring neurons in the auditory cortex had better synchronization (Eggermont and Smith, 1996).

Most neuron pairs with a peak shift displayed long discharge time differences (14 ms on average). This suggests that direct (i.e., monosynaptic) interaction was not obvious between nearby neurons. These results are consistent with the previous study in which there was no evidence indicating direct connection between CA1 pyramidal neurons nearby in the intracellular recordings in vitro (Nakajima et al., 1991).

\section{TASK-DEPENDENT SYNCHRONOUS ACTIVITY}

The four tasks used in the present study had different characteristics. In the RT/TC task, the pointer in the monitor was not presented and the monkeys always faced in a fixed direction. This indicated that the monkey could recognize same landmarks in each trial, and suggests that a cognitive map, in which spatial relationships of the landmarks are represented, is required for the performance of RT/ TC task. In the RT/P-TC task, however, they did not necessarily judge their position based on any cognitive map since the position was indicated by the pointer on the monitor. The VT (VT/P and VT/P-TC) tasks required the monkey to move a pointer to a target area on the monitor, and required no cognitive map system.

The task-dependent neural correlates in the HF were reported previously (Matsumura et al., 1999). In the present study, the synchronous activity in the $\mathrm{HF}$ and $\mathrm{PH}$ was also altered in a taskdependent manner with or without dependence on the firing rate. This task-dependent synchronous activity was most frequently found in the neuron pairs of pyramidal vs. pyramidal neurons, while the synchrony between interneurons was always task-independent. Furthermore, these neuron pairs with task-dependent CCGs displayed peak shift with positive peaks. These results suggest that information for specific tasks is encoded by the asymmetrical neural circuits connecting pyramidal neurons, and that interneurons are involved in task-independent common information across the tasks. Asymmetrical connection between pyramidal neurons

\section{A Peak polarity}
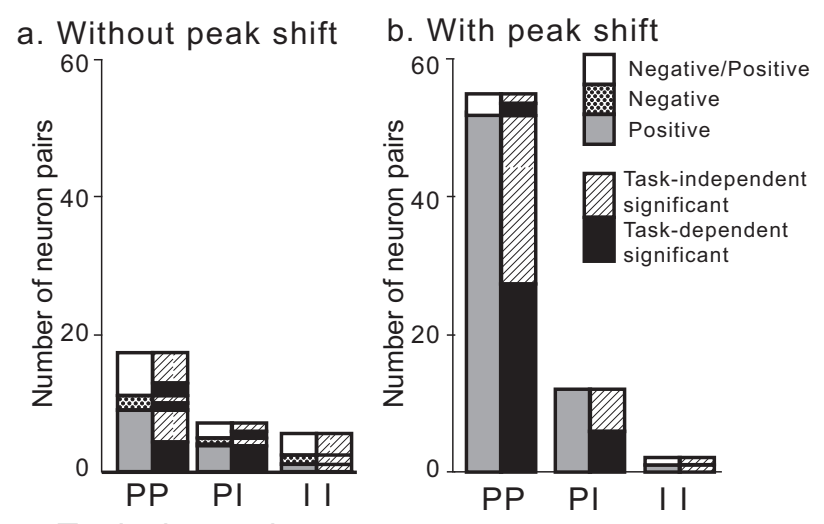

B Task-dependency

a. Without peak shift

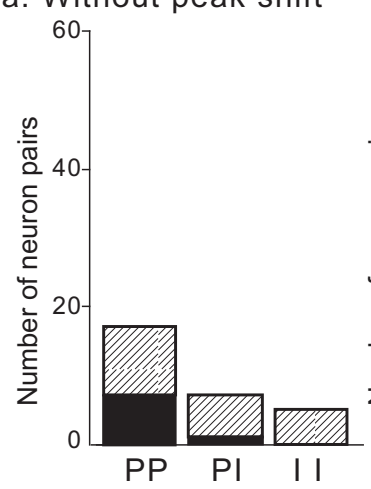

b. With peak shift

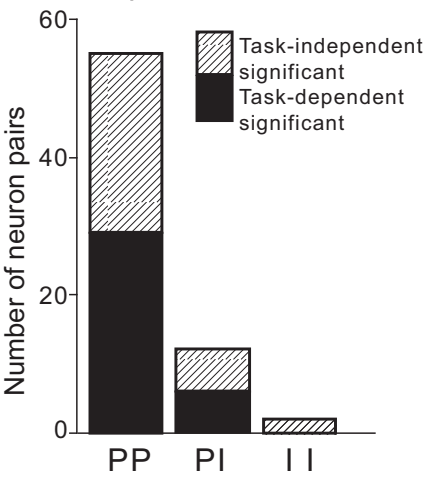

FIGURE 14 | Polarity (A) and task-dependency (B) of the CCG peaks without or with a peak shift in terms of the types of the neuron pairs. To facilitate comparison of the data between (A) (peak polarity) and (B) (task-dependency), corresponding task-dependency is shown in the right column of each type of neuron pair in (A). Note that most pairs with a peak shift had positive peaks. Positive, positive peaks; Negative, negative peaks; Negative/Positive, center-negative and surround-positive peaks. PP, pyramidal neuron vs. pyramidal neuron; PI, pyramidal neuron vs. interneuron; II, interneuron vs. interneuron. Task-independent significant, task-independent significant CCG peaks; task-dependent significant, task-dependent significant CCG peaks.

has been suggested to be a neurophysiological basis in sequence learning and memory in the rat HF (Skaggs et al., 1996; Lisman, 1999; Nadásdy et al., 1999; Sato and Yamaguchi, 2003; Wu and Yamaguchi, 2004). The present study further indicates that the asymmetrical neural circuits connecting pyramidal neurons are important in encoding of task-specific information as noted below, consistent with a computational study (Levy, 1996; Wagatsuma and Yamaguchi, 2007).

The task-dependent synchronous activity was found in both real and VT tasks, and there was no clear difference in the CCG peaks between the tasks. This result indicates that the task-dependent functional connectivity of the HF neurons may be produced in any kind of tasks. This also suggests that the monkey HF does not strictly contribute to spatial behaviors, but is involved in encoding of other factors such as task-specific information. It has been reported that about $40 \%$ of the monkey HF neurons 
Table 1 | Number of neuron pairs showing significant and non-significant cross-correlograms (CCGs) in each subregion of the HF and PH.

\begin{tabular}{llrrrrr}
\hline \multicolumn{7}{c}{ Type of neuron pairs } \\
\hline Subregion & Signif- & P vs. P & P vs. I & I vs. I & Subtotal & Total \\
& icant & & & & & \\
\hline CA1 & - & 23 & 3 & 0 & 26 & 84 \\
& + & 48 & 6 & 4 & 58 & \\
CA3 & - & 0 & 0 & 0 & 0 & 1 \\
& + & 1 & 0 & 0 & 1 & \\
DG & - & 5 & 15 & 5 & 25 & 47 \\
& + & 14 & 5 & 3 & 22 & \\
SUB & - & 8 & 0 & 2 & 10 & 20 \\
& + & 8 & 2 & 0 & 10 & \\
PH & - & 4 & 0 & 0 & 4 & 11 \\
& + & 7 & 0 & 0 & 7 & \\
Subtotal & - & 46 & 14 & 5 & 65 & \\
& + & 72 & 18 & 8 & 98 & \\
Total & + & 118 & 32 & 13 & 163 & \\
\hline
\end{tabular}

Pvs. P, Pyramidal vs. pyramidal neurons.

Pvs. I, Pyramidal neuron vs. interneuron.

I vs. I, Interneuron vs. interneuron.

$D G$, dentate gyrus; $S U B$, subiculum; $P H$, parahippocampal gyrus.

+ , significant $C C R ;-$, non-significant CCR.

Table 2 | Characteristics of the neuron pairs with significant CCRs in each subregion of the HF and PH regarding task-selectivity, peak shift, and peak polarity.

\begin{tabular}{|c|c|c|c|c|c|c|c|}
\hline & \multicolumn{2}{|c|}{ Task-selectivity } & \multicolumn{2}{|c|}{ Peak shift } & \multicolumn{3}{|c|}{ Peak polarity } \\
\hline & + & - & + & - & $\mathbf{P}$ & $\mathbf{N}$ & NP \\
\hline CA1 & 24 & 34 & 40 & 18 & 49 & 3 & 6 \\
\hline CA3 & 0 & 1 & 1 & 0 & 1 & 0 & 0 \\
\hline DG & 10 & 12 & 12 & 10 & 14 & 1 & 7 \\
\hline SUB & 4 & 6 & 10 & 0 & 9 & 0 & 1 \\
\hline $\mathrm{PH}$ & 5 & 2 & 6 & 1 & 6 & 1 & 0 \\
\hline
\end{tabular}

Each number indicates number of neuron pairs in each category. Task-selectivity (+), task-dependent CCR; task-selectivity (-), task-independent CCR; peak shift (+), CCRs with peak shift; peak shift (-), CCRs without peak shift; P, positive; N, negative; NP, center-negative and surround-positive peaks. Other descriptions as for Table 1.

showed place-differential activity, which were sensitive to tasks (i.e., task-dependent; Matsumura et al., 1999). The authors suggested that an assembly of reference frames may be a neural basis of episodic memory for various events. Consistently, rodent $\mathrm{HF}$ place cells were sensitive to specific reference frames (Samsonovich and McNaughton, 1997; Skaggs and McNaughton, 1998; Tabuchi et al., 2003). HF place cells in old rats displayed inconsistency to same reference frames, relating to cognitive and memory deficits (Barnes et al., 1997). The present finding of the task-dependent CCG peaks suggests that different neural circuits may be active for task-dependent memory. Taken together, it is strongly suggested that the HF neural circuits may be important for representation of specific information for the different tasks, and that neuronal populations selective to task situation or memory may originate in the HF.

\section{TASK-INDEPENDENT SYNCHRONOUS ACTIVITY}

All the three types of pairs (PP, PI, II) displayed task-independent CCG peaks. Data in Figures 6 and 7 suggest that the neuron pairs received common inputs as noted below. The positive and taskindependent CCG peaks were observed in the neuron pairs of pyramidal vs. pyramidal neurons (Figure 6) and pyramidal neuron vs. interneuron. In this case, common excitatory inputs for long duration ( $>300 \mathrm{~ms}$ ) could promote discharges of both types of neuron pairs. Negative CCG peaks shown in Figure 7 also suggest involvement of common inputs. This pattern was observed mainly in the neuron pairs of pyramidal neuron vs. interneuron. In addition, all pairs had no clear peak shift, and CCG in all pairs except one were task-independent. The possible circuits might involve inhibitory inputs from upstream inhibitory neurons that could suppress activity of both a pyramidal neuron and an interneuron. Center-negative surround-positive CCG peaks (Figure 8) were observed in all three types of neuron pairs. The majority of the pairs had no clear peak shift and their peaks were task-independent. More complex neural circuits including the inhibitory inputs could be involved in this case.

The GABAergic innervation from the medial septum and the nucleus of the diagonal band, which is a major source of theta rhythm in the HF, inhibits or disinhibits the pyramidal neurons through interneurons within the HF (Stewart and Fox, 1990). The cholinergic system from the same brain regions excites directly the pyramidal neurons or interneurons (Stewart and Fox, 1990). These inhibition and excitation mechanisms in the HF might contribute to form CCG peaks without a peak shift implicating common inputs. The common inputs might convey task-independent information such as arousal and intention, which were supposed to be similar across the tasks, since behavioral requirement (i.e., joystick manipulation), reward (juice), and behavioral performance were similar across the tasks. It has been reported that there are several types of interneurons in the HF based on the morphology and pharmacology (Freund and Buzsáki, 1996; Halasy et al., 1996). Unidirectional and reciprocal inhibitory interneurons connect with pyramidal neurons (Stewart and Fox, 1990). The local interneurons within the HF could modulate discharge patterns to generate complex CCG peaks as well as oscillations in the ACRs. Further studies are necessary to elucidate the neural circuits in the HF.

\section{THETA OSCILLATION AND TRIGGER-LIKE ACTIVITY}

Oscillatory activity was involved in the synchronization between the HF neurons in primates (Figure 9 and weakly in Figures 4 and 5), consistent with the data in rodents (Mizumori et al., 1990; Skaggs et al., 1996; Tabuchi et al., 2000). However, there were some differences between primate and rodent data. First, frequency of the theta oscillation was low at $3 \mathrm{~Hz}$ in the present study. This frequency is consistent with the electroencephalogram (EEG) data at $3-12 \mathrm{~Hz}$ in monkeys (Stewart and Fox, 1990) and humans (Kahana et al., 1999), but lower than $9 \mathrm{~Hz}$ in rodents (Tabuchi et al., 1999). Second, theta oscillation was not common in monkeys (3/98 pairs in the present study). Third, theta rhythm was observed only between 
the pyramidal neurons. These data are inconsistent with those in rats in which the theta oscillation was frequently found in both pyramidal neurons and interneurons (Mizumori et al., 1990; Skaggs et al., 1996; Tabuchi et al., 1999). These suggest different roles of theta oscillation between primates and rodents.

In three pairs of pyramidal vs. pyramidal neurons, discharges of one neuron were followed by those of the other neurons with high frequency burst (Figure 10). This indicates that the pyramidal neurons of these three pairs can be separated into two types; trigger type neurons with low frequent discharges and bursting neurons. The CCRs for these pairs were task-dependent. The bursting neurons also displayed place-differential responses. These results suggest that these neuron types might be involved in encoding of task-dependency by induction of long-term potentiation in which high frequency activation plays an important role.

\section{CONCLUSION}

In the present study cross-correlation was examined between two neurons simultaneously recorded during performance of the four spatial tasks. The results indicate that functional connectivity of some HF neuron pairs changed in a task-dependent manner. These task-dependent CCRs were frequently observed in the pairs

\section{REFERENCES}

Abrahams, S., Pickering, A., Polkey, C. E., and Morris, R. G. (1997). Spatial memory deficits in patients with unilateral damage to the right hippocampal formation. Neuropsychologia 35, 11-24.

Aertsen,A.M.H. J., Gerstein, G. L., Habib, M. K., and Palm, G. (1989). Dynamics of neuronal firing correlation: modulation of "effective connectivity." J. Neurophysiol. 61, 900-917.

Aguirre, G. K., and D'Esposito, M. (1997). Environmental knowledge is subserved by separable dorsal/ ventral neural areas. J. Neurosci. 17, 2512-2518.

Aguirre, G. K., Detre, J. A., Alsop, D. C., and D'Esposito, M. (1996). The parahippocampus subserves topographical learning in man. Cereb. Cortex 6, 823-829.

Aika, Y., Ren, J. Q., Kosaka, K., and Kosaka, T. (1994). Quantitative analysis of GABA-like-immunoreactive and parvalbumin-containing neurons in the CAl region of the rat hippocampus using a stereological method, the dissector. Exp. Brain Res. 99, 267-276.

Barnes, C. A., Suster, M. S., Shen, J., and McNaughton, B. L. (1997). Multistability of cognitive maps in the hippocampus of old rats. Nature 388, 272-275.

Bieber, S. L., and Smith, D. V. (1986). Multivariate analysis of sensory data: a comparison of methods. Chem. Senses 11, 19-47.

Breese, C. R., Hampson, R. E., and Deadwyler, S.A. (1989). Hippocampal

of pyramidal vs. pyramidal neurons. The time difference of their synchronous discharges suggested indirect asymmetrical connections between these neurons. These results support the idea that task-dependent neuronal networks in the HF encode context or reference frames, which might be a neural basis for episodic memory. In contrast, the task-independent CCRs were observed in all three types of neuron pairs. This suggests involvement of non-selective common inputs across the tasks such as arousal or intentional inputs to both pyramidal neurons and interneurons in the HF. These results suggest that the HF task-dependent neural circuits with asymmetrical connections are critical to disambiguously guide animals to places associated with reward in different situations.

\section{ACKNOWLEDGMENTS}

This work was supported partly by CREST, JST, Japan, JSPS Asian Core Program, and the Ministry of Education, Science, Sports, and Culture, Grant-in-Aid for Scientific Research (A; 22240051). The authors wish to thank Dr. Kari Hoffman (York University) and Dr. Bruce L. McNaughton (University of Lethbridge) for help in preparing the manuscript, and Dr. Gyorgy Buzsaki (the State University of New Jersey) and Dr. Sidney I. Wiener (Collège de France, Paris) for reviewing this manuscript.

place cells: stereotypy and plasticity. J. Neurosci. 9, 1097-1111.

Clayton, N. S. (1995). Development of memory and the hippocampus: comparison of food-storing and non-storing birds on a one-trial associative memory task. J. Neurosci. 15, 2796-2807.

Csicsvari, J., Hirase, H., Czurkó, A., and Buzsáki, G. (1998). Reliability and state dependence of pyramidal cell-interneuron synapses in the hippocampus: an ensemble approach in the behaving rat. Neuron 21, 179-189. Csicsvari, J., Hirase, H., Czurkó, A., Mamiya, A., and Buzsáki, G. (1999). Oscillatory coupling of hippocampal pyramidal cells and interneurons in the behaving rat. J. Neurosci. 19, 274-287.

Dayawansa, S., Kobayashi, T., Hori, E., Umeno, K., Tazumi, T., Ono, T., and Nishijo, H. (2006). Conjunctive effects of reward and behavioral episodes on hippocampal place-differential neurons of rats on a mobile treadmill. Hippocampus 16, 586-595.

Eggermont, J. J., and Smith, G. M. (1996). Neural connectivity only accounts for a small part of neural correlation in auditory cortex. Exp. Brain Res. 110, 379-391.

Eichenbaum, H., Kuperstein, M., Fagan, A., and Nagode, J. (1987). Cuesampling and goal-approach correlates of hippocampal unit activity in rats performing an odor-discrimination task. J. Neurosci. 7, 716-732.

Eifuku, S., Nishijo, H., Kita, T., and Ono, T. (1995). Neuronal activity in the primate hippocampal formation during a conditional association task based on the subject's location. J. Neurosci. 15, 4952-4969.

Fox, S. E., and Ranck, J. B. J. (1981). Electrophysiological characteristics of hippocampal complex- spike cells and theta cells. Exp. Brain Res. 41, 399-410.

Frank, L. M., Brown, E. N., and Wilson, M. (2000). Trajectory encoding in the hippocampus and entorhinal cortex. Neuron 27, 169-178.

Freund, T. F., and Buzsáki, G. (1996). Interneurons of the hippocampus. Hippocampus 6, 347-470.

Gallo, M. (2005). Hippocampus, temporal context and taste memories. Chem. Senses 30, 160-161.

Ghaem, O., Mellet, E., Crivello, F., Tzourio, N., Mazoyer, B., Berthoz, A., and Denis, M. (1997). Mental navigation along memorized routes activates the hippocampus, precuneus, and insula. Neuroreport 8, 739-744.

Gochin, P. M., Kaltenbach, J. A., and Gerstein, G. (1989). Coordinated activity of neuron pairs in anesthetized rat dorsal cochlear nucleus. Brain Res. 497, 1-11.

Halasy, K., Buhl, E. H., Lörinczi, Z., Tamás, G., and Somogyi, P. (1996). Synaptic target selectivity and input of GABAergic basket and bistratified interneurons in the CA1 area of the rat hippocampus. Hippocampus 6, 306-329.

Hampton, R. R., Hampstead, B. M., and Murray, E. A. (2004). Selective hippocampal damage in rhesus monkeys impairs spatial memory in an openfield test. Hippocampus 14, 808-818.
Hirase, H., Leinekugel, X., Csicsvari, J., Czurkó, A., and Buzsáki, G. (2001). Behavior-dependent states of the hippocampal networkaffect functional clustering of neurons. J. Neurosci.21, RC145.

Ho, A. S., Hori, E., Nguyen, P. H. T., Urakawa, S., Kondoh, T., Torii, K., Ono, T., and Nishijo, H. (2011). Hippocampal neuronal responses during signaled licking of gustatory stimuli in different contexts. Hippocampus 21, 502-519.

Hori, E., Tabuchi, E., Matsumura, N., Tamura, R., Eifuku, S., Endo, S., Nishijo, H., and Ono, T. (2003). Representation of place by monkey hippocampal neurons in real and virtual translocation. Hippocampus 13, 148-154.

Ito, R., Robbins, T. W., McNaughton, B. L., and Everitt, B. J. (2006). Selective excitotoxic lesions of the hippocampus and basolateral amygdala have dissociable effects on appetitive cue and place conditioning based on path integration in a novel Y-maze procedure. Eur. J. Neurosci. 23, 3071-3080.

Ito, R., Robbins, T. W., Pennartz, C. M., and Everitt, B. J. (2008). Functional interaction between the hippocampus and nucleus accumbens shell is necessary for the acquisition of appetitive spatial context conditioning. J. Neurosci. 28, 6950-6959.

Jog, M. S., Kubota, Y., Connolly, C. I., Hillegaart, V., and Graybiel, A. M. (1999). Building neural representations of habits. Science 286, 1745-1749.

Jung,M.W.,Wiener,S.I., and McNaughton, B. L. (1994). Comparison of spatial firing characteristics of units in dorsal 
and ventral hippocampus of the rat. J. Neurosci. 14, 7347-7356.

Kahana, M. J., Sekuler, R., Caplan, J. B., Kirschen, M., and Madsen, J.R. (1999). Human theta oscillations exhibit task dependence during virtual maze navigation. Nature 399, 781-784.

Kennedy, P. J., and Shapiro, M. L. (2009). Motivational states activate distinct hippocampal representations to guide goal-directed behaviors. Proc. Natl. Acad. Sci. U.S.A. 106, 10805-10810.

Kobayashi, T., Nishijo, H., Fukuda, M., Bures, J., and Ono, T. (1997). Task-dependent representations in rat hippocampal place neurons. $J$. Neurophysiol. 78, 597-613.

Levy, W. B. (1996). A sequence predicting $\mathrm{CA} 3$ is a flexible associator that learns and uses context to solve hippocampallike tasks. Hippocampus 6, 579-590.

Lisman, J.E. (1999). Relating hippocampal circuitry to function: recall of memory sequences by reciprocal dentate-CA3 interactions. Neuron 22, 233-242.

Maguire, E. A., Burke, T., Phillips, J., and Staunton, H. (1996a). Topographical disorientation following unilateral temporal lobe lesions in humans. Neuropsychologia 34, 993-1001.

Maguire, E. A., Frackowiak, R. S. J., and Frith, C. D. (1996b). Learning to find your way: a role for the human hippocampal formation. Proc. Biol. Sci. $263,1745-1750$.

Maguire, E. A., Frackowiak, R. S. J., and Frith, C. D. (1997). Recalling routes around London: activation of the right hippocampus in taxi drivers. J. Neurosci. 15, 7103-7110.

Maldonado, P. E., and Gerstein, G. L. (1996). Neuronal assembly dynamics in the rat auditory cortex during reorganization induced by intracortical microstimulation. Exp. Brain Res. $112,431-441$.

Matsumura, N., Nishijo, H., Tamura, R., Eifuku, S., Endo, S., and Ono, T. (1999). Spatial- and task-dependent neuronal responses during real and virtual translocation in the monkey hippocampal formation. J. Neurosci. 19, 2381-2393.

McNaughton, B. L., Barnes, C. A., and O'Keefe, J. (1983). The contribution of position, direction, and velocity to single unit activity in the hippocampus of freely-moving rats. Exp. Brain Res. 52, 41-49.

Mellet, E., Briscogne, S., Tzourio-Mazoyer, N., Ghaem, O., Petit, L., Zago, L., Etard, O., Berthoz, A., Mazoyer, B., and Denis, M. (2000). Neural correlates of topographic mental exploration: the impact of route versus survey perspective learning. Neuroimage 12, 588-600.

Mizumori, S. J. Y., Barnes, C. A., and McNaughton, B. L. (1990). Behavioral correlates of theta-on and theta-off cells recorded from hippocampal formation of mature young and aged rats. Exp. Brain Res. 80, 365-373.

Morris, R. G., Garrud, P., Rawlins, J. N. P., and O'Keefe, J. (1982). Place navigation impaired in rats with hippocampal lesions. Nature 297, 681-683.

Muller, R. U., and Kubie, J. L. (1987). The effects of changes in the environment on the spatial firing of hippocampal complex-spike cells. J. Neurosci. 7 , 1951-1968.

Muller, R. U., Kubie, J. L., and Ranck, J. B. Jr. (1987). Spatial firing patterns of hippocampal complex-spike cells in a fixed environment. J. Neurosci. 7, 1935-1950.

Munk, M. H. J., Nowak, L. G., Nelson, J. I., and Bullier, J. (1995). Structural basis of cortical synchronization. II. Effects of cortical lesions. J. Neurophysiol. 74, 2401-2414.

Nadásdy, Z., Hirase, H., Czurkó, A., Csicsvari, J., and Buzsaki, G. (1999). Relay and time compression of recurring spike sequences in the hippocampus. J. Neurosci. 19, 9497-9507.

Nakajima, S., Frank, J. E., Bilkey, D., and Schwartzkroin, P. A. (1991). Local circuit synaptic interactions between CA1 pyramidal cells and interneurons in the kainate-lesioned hyperexcitable hippocampus. Hippocampus 1, 67-78.

Nishijo, H., and Norgren, R. (1990). Responses from parabrachial gustatory neurons in behaving rats. $J$. Neurophysiol. 63, 707-724.

Nishijo, H., and Norgren, R. (1991). Parabrachial gustatory neural activity during licking by rats. J. Neurophysiol. 66, 974-985.

Nishijo, H., Ono, T., Eifuku, S., and Tamura, R. (1997). The relationship between monkey hippocampus placerelated neural activity and action in space. Neurosci. Lett. 226, 57-60.

Nishijo, H., Ono, T., Tamura, R., and Nakamura, K. (1993). Amygdalar and hippocampal neuron responses related to recognition and memory in monkey. Prog. Brain Res. 95, 339-357.

Nowak, L. G., Munk, M. H. J., Nelson, J. I., James, A. C., and Bullier, J. (1995). Structural basis of cortical synchronization. I. Three types of interhemispheric coupling. J. Neurophysiol. 74, 2379-2400.

O'Keefe, J., and Dostrovsky, J. (1971). The hippocampus as a spatial map. Preliminary evidence from unit activity in the freely-moving rat. Brain Res. $34,171-175$.

O'Keefe, J., and Nadel, L. (1978). The Hippocampus as a Cognitive Map. Oxford: Clarendon Press.

O’Mara, S. M., Rolls, E. T., Berthoz, A., and Kesner, R. P. (1994). Neurons responding to whole-body motion in the primate hippocampus. J. Neurosci. 14, 6511-6523.

Ono, T., Nakamura, K., Nishijo, H., and Eifuku, S. (1993). Monkey hippocam- pal neuron related to spatial and nonspatial functions. J. Neurophysiol. 70, 1516-1529.

Perkel, D. H., Gerstein, G. L., and Moore, G. P. (1967). Neuronal spikes trains and stochastic point processes. I. The single spike train. Biophys. J. 7, 391-418.

Redish, A. D., and Touretzky, D. S. (1997). Cognitive maps beyond the hippocampus. Hippocampus 7, 15-35.

Rolls, E. T., and Xiang, J.-Z. (2005) Reward-spatial view representations and learning in the primate hippocampus. J. Neurosci. 25,6167-6174.

Sakurai, Y. (1996). Hippocampal and neocortical cell assemblies encode memory processes for different types of stimuli in the rat. J. Neurosci. 16 , 2809-2819.

Samsonovich, A., and McNaughton, B. L. (1997). Path integration and cognitive mapping in a continuous attractor neural network model. J. Neurosci. 17, 5900-5920.

Sato, N., and Yamaguchi, Y. (2003) Memory encoding by theta phase precession in the hippocampal network. Neural Comput. 15, 2379-2397.

Skaggs, W. E., and McNaughton, B. L. (1998). Spatial firing properties of hippocampal CA1 populations in an environment containing two visually identical regions. J. Neurosci. 18 , 8455-8466.

Skaggs, W. E., McNaughton, B. L., Permenter, M.,Archibeque, M., Vogt, J., Amaral,D.G., and Barnes, C. A. (2007) EEG sharp waves and sparse ensemble unit activity in the macaque hippocampus. J. Neurophysiol. 98, 898-910.

Skaggs, W.E., McNaughton, B. L., Wilson, M. A., and Barnes, C. A. (1996). Theta phase precession in hippocampal neuronal populations and the compression of temporal sequences. Hippocampus 6, 149-172.

Stewart, M., and Fox, S. E. (1990). Do septal neurons pace the hippocampal theta rhythm? Trends. Neurosci. 13 163-169.

Suzuki, W.A., Miller,E. K., and Desimone, R. (1997). Object and place memory in the macaque entorhinal cortex. $J$. Neurophysiol. 78, 1062-1081.

Tabuchi, E., Mulder, A. B., and Wiener, S. I. (1999). Dorsal and ventral hippocampal neuronal responses in a differentially rewarded task. Abstr. Soc. Neurosci. 25, 558.4 .

Tabuchi, E., Mulder, A. B., and Wiener, S. I. (2000). Position and behavioral modulation of synchronization of hippocampal and accumbens neuronal discharges in freely moving rats. Hippocampus 10, 717-728.

Tabuchi, E., Mulder, A. B., and Wiener, S. I. (2003). Reward value invariant place responses and reward site associated activity in hippocampal neurons of behaving rats. Hippocampus 13 , 117-132.

Takahashi, N., Kawamura, M., Shiota, J., Kasahata, N., and Hirayama, K. (1997). Pure topographic disorientation due to right retrosplenial lesion. Neurology 49, 464-469.

Takahashi, S., and Sakurai, Y. (2009). Sub-millisecond firing synchrony of closely neighboring pyramidal neurons in hippocampal cal of rats during delayed non-matching to sample task. Front Neural Circuits 3:9. doi: 10.3389/ neuro.04.009.2009

Thorndyke, P. W., and Hayes-Roth, B. (1982). Difference in spatial knowledge acquired from maps and navigation. Cogn. Psychol. 14, 560-589.

Wagatsuma, H., and Yamaguchi, Y. (2007). Neural dynamics of the cognitive map in the hippocampus. Cogn. Neurodyn. $1,119-141$.

Wiebe, S. P., and Stäubli, U. V. (2001). Recognition memory correlates of hippocampal theta cells. J. Neurosci. 21, 3955-3967.

Wood, E. R., Dudchenko, P. A., Robitsek, R. J., and Eichenbaum, H. (2000). Hippocampal neurons encode information about different types of memory episodes occurring in the same location. Neuron 27, 623-633.

Woodson, W., Nitecka, L., and BenAri, Y. (1989). Organization of the GABAergic system in the rat hippocampal formation: a quantitative immunocytochemical study. J. Comp. Neurol. 280, 254-271.

$\mathrm{Wu}, \mathrm{Z}$., and Yamaguchi, Y. (2004). Inputdependent learning rule for the memory of spatiotemporal sequences in hippocampal network with theta phase precession. Biol. Cybern. 90, 113-124.

Conflict of Interest Statement: The authors declare that the research was conducted in the absence of any commercial or financial relationships that could be construed as a potential conflict of interest.

Received: 11 January 2011; accepted: 24 June 2011; published online: 14 July 2011. Citation: Hori E, Tabuchi E, Matsumura N, Ono T and Nishijo H (2011) Taskdependent and independent synchronous activity of monkey hippocampal neurons in real and virtual translocation. Front. Behav. Neurosci. 5:36. doi: 10.3389/ fnbeh.2011.00036

Copyright (c) 2011 Hori, Tabuchi, Matsumura, Ono and Nishijo. This is an open-access article subject to a nonexclusive license between the authors and Frontiers Media SA, which permits use, distribution and reproduction in other forums, provided the original authors and source are credited and other Frontiers conditions are complied with 